INSTITUT NATIONAL DE RECHERCHE EN INFORMATIQUE ET EN AUTOMATIQUE

\title{
Deconvolution for the Wasserstein Metric and Geometric Inference
}

Claire Caillerie — Frédéric Chazal — Jérôme Dedecker — Bertrand Michel

\section{$\mathbf{N}^{\circ} 7678$}

Juillet 2011

_ Algorithms, Certification, and Cryptography _ 



\title{
Deconvolution for the Wasserstein Metric and Geometric Inference
}

\author{
Claire Caillerie ${ }^{*}$, Frédéric Chazal †, Jérôme Dedecker f $^{\dagger}$, Bertrand \\ Michel \$ \\ Theme : Algorithms, Certification, and Cryptography \\ Équipe-Projet Geometrica \\ Rapport de recherche $\mathrm{n}^{\circ} 7678$ - Juillet $2011-28$ pages
}

\begin{abstract}
Recently, [4 have defined a distance function to measures to answer geometric inference problems in a probabilistic setting. According to their result, the topological properties of a shape can be recovered by using the distance to a known measure $\nu$, if $\nu$ is close enough to a measure $\mu$ concentrated on this shape. Here, close enough means that the Wasserstein distance $W_{2}$ between $\mu$ and $\nu$ is sufficiently small. Given a point cloud, a natural candidate for $\nu$ is the empirical measure $\mu_{n}$. Nevertheless, in many situations the data points are not located on the geometric shape but in the neighborhood of it, and $\mu_{n}$ can be too far from $\mu$. In a deconvolution framework, we consider a slight modification of the classical kernel deconvolution estimator, and we give a consistency result and rates of convergence for this estimator. Some simulated experiments illustrate the deconvolution method and its application to geometric inference on various shapes and with various noise distributions.
\end{abstract}

Key-words: Deconvolution, Wasserstein distance, geometric inference, computational topology

\footnotetext{
* INRIA Saclay

$\dagger$ INRIA Saclay

¥ Laboratoire MAP5, UMR CNRS 8145 Université Paris Descartes

$\S$ Laboratoire de Statistique Théorique et Appliquée, Université Pierre et Marie Curie -
} Paris 6

Centre de recherche INRIA Saclay - Île-de-France

Parc Orsay Université

4, rue Jacques Monod, 91893 ORSAY Cedex

Téléphone : +33172925900 


\section{Déconvolution pour la métrique de Wasserstein et inférence géométrique}

Résumé : La notion de fonction distance à une mesure récemment introduite dans [4] permet de répondre à des problèmes d'inférence géométrique dans un cadre probabiliste : les propriétés topologiques d'un compact $K \subset \mathbb{R}^{d}$ peuvent être estimées à l'aide de la fonction distance à une mesure de probabilité connue $\nu$ si celle-ci se trouve suffisamment proche (au sens de la distance de Wasserstein $W_{2}$ ) d'une mesure $\mu$ dont $K$ est le support. En pratique lorsque les observations sont corrompues par du bruit, la mesure empirique associée aux observations n'est généralement pas assez proche de $\mu$ pour pouvoir être utilisée directement. Dans cet article, on propose une solution à ce problème en considérant un modèle de convolution pour lequel la loi du bruit est supposée connue. On considère une variante de l'estimateur par noyau de déconvolution classique dont on établit la consistence et des vitesses de convergence. On illustre la méthode proposée et ses applications en inférence géométrique sur différentes formes géométriques et différentes distributions de bruit sur les observations.

Mots-clés : Déconvolution, Distance de Wasserstein, Inférence géométrique, Topologie algorithmique 


\section{Introduction}

Infering topological and geometric information from multivariate data is a problem which is attracting a lot of interest since a couple of decades. Many statistical methods have been developed to model and estimate geometric features from point cloud data that are usually considered as independent observations drawn according to a common distribution $\mu$ in an Euclidean space $\mathbb{R}^{d}$. In low dimensions, principal curves and principal surfaces have been early proposed by [17. to study simple manifolds. More elaborated structures can be also studied with density-based methods. For instance, filament estimation has been the subject of several works, see for instance [13] and [14 for recent contributions. In a more general context, set estimation deals with problems in the interplay between statistics and geometry. This field includes estimation of supports, boundaries and level sets, see $[8$ for a large overview on this topic. Cluster analysis algorithms also provide geometric information. One popular approach of clustering proposed by [16] consists in defining clusters as connected components of the levels sets associated to a density $f$, see for instance 7 and $[1$. Another statistical work by [19] proposes estimators of the entropy dimension of the support of $\mu$ and of the number of clusters of the support in the case of corrupted data. The paper of [9] addresses estimation of the surface area of a $d$-dimensional body, as defined by the Minkowski measure. These above mentioned works propose efficient statistical methods for geometric inference but they usually do not provide topological guarantees on the estimated geometric quantities.

On the other hand many non stochastic methods have been proposed in computational geometry to infer the geometry of an unknown object from a set of data point sampled around it. In this context, distance functions to the data have shown to be efficient tools to robustly infer precise information about the geometry of the object. More precisely, [5] and [3] show that the sublevel sets of the distance function to the data can be used to recover the geometry of the unknown object. These methods offer strong geometric and topological guarantees but they rely on strong sampling assumptions that usually do not apply in a statistical framework. In particular, they fail when applied on data corrupted by outliers.

Recently, some efforts have been made to bridge the gap between the statistical and geometric approaches. For example, assuming that the observations are independently drawn from a probability measure that is the convolution of the uniform measure on a submanifold $M$ with a Gaussian noise measure supported by the normals to $M, 23$ propose an algorithm to recover the Betti numbers of $M$. A major limitation of this method is that the noise should verify a strong variance condition.

In a different perspective [4 have generalized the approach of [3] by extending the notion of distance function from compact sets to probability measures. This new framework allows to robustly infer geometric properties of a distribution $\mu$ using independent observations drawn according to a distribution $\mu^{\prime}$ "close" to $\mu$ where the closeness between probability distributions is assessed by a Wasserstein distance $W_{p}$ defined by

$$
W_{p}\left(\mu, \mu^{\prime}\right)=\inf _{\pi \in \Pi\left(\mu, \mu^{\prime}\right)}\left(\int_{\mathbb{R}^{d} \times \mathbb{R}^{d}}\|x-y\|^{p} \pi(d x, d y)\right)^{\frac{1}{p}}
$$


where $\Pi\left(\mu, \mu^{\prime}\right)$ is the set of probability measures on $\mathbb{R}^{d} \times \mathbb{R}^{d}$ that have marginals $\mu$ and $\mu^{\prime},\|$.$\| is a norm and p \geq 1$ is a real number (see [26] or [28]).

Given a probability distribution $\mu$ in $\mathbb{R}^{d}$ and a real parameter $0 \leq m \leq 1$, [4] generalize the notion of distance to the support of $\mu$ by the function $\delta_{\mu, m}$ : $x \in \mathbb{R}^{d} \mapsto \inf \{r>0: \mu(B(x, r))>m\}$ where $B(x, r)$ is the closed Euclidean ball of center $x$ and radius $r$. To avoid issues due to discontinuities of the map $\mu \mapsto \delta_{\mu, m}$, the distance function to $\mu$ with paprameter $m_{0} \in[0,1]$ is defined by

$$
\mathrm{d}_{\mu, m_{0}}: \mathbb{R}^{d} \rightarrow \mathbb{R}^{+}, x \mapsto \sqrt{\frac{1}{m_{0}} \int_{0}^{m_{0}}\left(\delta_{\mu, m}(x)\right)^{2} d m} .
$$

The function $\mathrm{d}_{\mu, m_{0}}$ shares many properties with classical distance functions that make it well-suited for geometric inference purposes. In particular, the map $\mu \mapsto \mathrm{d}_{\mu, m_{0}}$ is $1 / \sqrt{m_{0}}$-Lipschitz, i.e.

$$
\sup _{x \in \mathbb{R}^{d}}\left|\mathrm{~d}_{\mu, m_{0}}(x)-\mathrm{d}_{\mu^{\prime}, m_{0}}(x)\right|=\left\|\mathrm{d}_{\mu, m_{0}}-\mathrm{d}_{\mu^{\prime}, m_{0}}\right\|_{\infty} \leq \frac{1}{\sqrt{m_{0}}} W_{2}\left(\mu, \mu^{\prime}\right) .
$$

This property ensures that the distance functions associated to close measures (for the $W_{2}$ metric) have close sublevel sets. Moreover, the function $\mathrm{d}_{\mu, m_{0}}^{2}$ is semiconcave (i.e. $x \mapsto\|x\|^{2}-\mathrm{d}_{\mu, m_{0}}^{2}(x)$ is convex) ensuring strong regularity properties on the geometry of its sublevel sets - see [24] for more informations on the geometric properties of semiconcave functions. Using these properties 44 (Corollary 4.11) prove, under some general assumptions, that if $\mu^{\prime}$ is a probability distribution approximating $\mu$, then the sublevel sets of $\mathrm{d}_{\mu^{\prime}, m_{0}}$ provide a topologically correct approximation of the support of $\mu$. The statement of such a result requires the following definitions. A probability measures is said to have dimension at most $k>0$ if there exits a constant $C(\mu)$ such that for any point $x$ in the support of $\mu$ and any sufficiently small $\varepsilon>0$ one has $\mu(B(x, \varepsilon)) \geq C(\mu) \varepsilon^{k}$. Given a compact set $G \subset \mathbb{R}^{d}$ and a real number $\alpha>0$ the $\alpha$-reach of $G$ denoted $\operatorname{reach}_{\alpha}(G)$ is a geometric quantity related to the critical points of the (classical) distance function $\mathrm{d}_{G}$ to $G, \mathrm{~d}_{G}(x)=\inf \{d(x, y) y \in G\}$, that provides a measure of the regularity of $G$ (see [3] for the definition).

Theorem 1. Let $\mu$ be a measure that has dimension at most $k>0$ with compact support $G$ such that reach ${ }_{\alpha}(G) \geq R>0$ for some $\alpha>0$. Let $\nu$ be another measure and $\varepsilon$ be an upper bound on the uniform distance between $\mathrm{d}_{G}$ and $\mathrm{d}_{\nu, m_{0}}$. Then, for any $r \in\left[4 \varepsilon / \alpha^{2}, R-3 \varepsilon\right]$ and any $\left.\eta \in\right] 0, R[$, the $r$-sublevel sets of $\mathrm{d}_{\mu, m_{0}}$ and the $\eta$-sublevel sets of $\mathrm{d}_{G}$ are homotopy equivalent as soon as:

$$
W_{2}(\mu, \nu) \leq \frac{R \sqrt{m_{0}}}{5+4 / \alpha^{2}}-C(\mu)^{-1 / k} m_{0}^{1 / k+1 / 2} .
$$

Roughly speaking, this result means that if ones knows a measure $\nu$ that is close to $\mu$ for the Wasserstein metric, the level sets of $d_{\nu, m_{0}}$ can be used to infer the topology of the sublevel sets of the distance function to the support $G$ of $\mu$. In practice if one observes a set of points independently sampled according to the distribution $\mu$ (resp. to some distribution $\mu^{\prime}$ that is close to $\mu$ ), a natural candidate for $\nu$ is the empirical measure of the points cloud $\mu_{n}$. Indeed, $\mathbb{E}\left(W_{2}^{2}\left(\mu_{n}, \mu\right)\right)$ (resp $\left.\mathbb{E}\left(W_{2}^{2}\left(\mu_{n}, \mu^{\prime}\right)\right)\right)$ converges to zero as $n$ tends to infinity, as shown by [18]. 
However, in many situations the data is contaminated by noise, namely we observe some points drawn according to the convolution $\mu \star \nu$ where $\mu$ is supported by the unknown compact set $G$ and $\nu$ is the distribution of the noise. In such a situation, $\mathbb{E}\left(W_{2}^{2}\left(\mu_{n}, \mu\right)\right)$ does not converges to zero anymore, and $\mu_{n}$ may be too far from $\mu$ to apply Theorem 1. The aim of this article is to propose a deconvolution estimator $\hat{\mu}_{n}$ close to $\mu$ for the Wasserstein metric, and then to use the levels sets of $\mathrm{d}_{\hat{\mu}_{n}, m_{0}}$ to infer the topology of the sublevel sets of the distance function to $G$.

Many papers deal with the convolution model from a statistical point of view. We focus here on works related to support estimation or geometric inference. Support estimation in the convolution setting has been the subject of recent works mostly in the univariate case. In [15] and [10, the boundary of the support is detected via the large values of the derivate of the density estimator under the assumption that the density of $\mu$ has a discontinuity at the boundary. An alternative method based on moment estimation is proposed by [22] without assuming that the density is discontinuous at the boundary. For the multivariate case, 21] proposes an estimator of the support based on a resampling strategy, that satisfy some consistency properties. Still in the convolution setting, [19] gives estimates of the entropy dimension of the support $G$ and of the number of clusters of $G$.

In this paper, we study the behavior of a deconvolution estimator with respect to the Wassertein metric $W_{2}$. In the applications we have in mind, $\mu$ is typically supported by a submanifold of $\mathbb{R}^{d}$ with dimension strictly less than $d$. Consequently, we shall not assume that $\mu$ has a density with respect to the Lebesgue measure on $\mathbb{R}^{d}$. In fact, except that it is compactly supported, we shall make no further assumptions on $\mu$.

Besides the geometric applications we have in mind, studying the properties of probability estimators for the $W_{2}$ metric is also interesting in itself. Firstly, contrary to the $\mathbb{L}_{p}$-distances between probability densities (except for $p=1$, which conincides with the total variation distance), the distances $W_{p}$ are true distances between probability distributions. Secondly, many natural estimators $\hat{\mu}_{n}$ of $\mu$ are singular with respect to $\mu$ (think of the empirical measure in most cases), and consequently the total variation distance between $\hat{\mu}_{n}$ and $\mu$ is equal to 2 for any $n$. This is the case of our deconvolution estimator, if the support $G$ is a submanifold in $\mathbb{R}^{d}$ with dimension srictly less than $d$. Wasserstein metrics appear as natural distances to evaluate the performance of such estimators.

The first section of this paper is devoted to the theoretical aspects of the paper. We first define the deconvolution estimator $\hat{\mu}_{n}$ and then we give rates of convergence for $\mathbb{E}\left(W_{2}^{2}\left(\hat{\mu}_{n}, \mu\right)\right)$. The second section presents some numerical experiments with applications to geometric inference.

\section{Deconvolution for the Wasserstein metric}

We start with some notation. The inner product $<\cdot, \cdot>$ from $\mathbb{R}^{d} \times \mathbb{R}^{d}$ to $\mathbb{R}$ is defined as follows: for $x=\left(x_{1}, \ldots, x_{d}\right)^{t}$ and $y=\left(y_{1}, \ldots, y_{d}\right)^{t},\langle x, y>=$ $x_{1} y_{1}+\cdots+x_{d} y_{d}$. The euclidean norm of $x$ is denoted by $\|x\|=\sqrt{\langle x, x\rangle}$. 
In the following, we denote by $\mu^{*}$ (respectively $f^{*}$ ) the Fourier transform of the probability measure $\mu$ (respectively of the integrable function $f$ ), that is:

$$
\mu^{*}(x)=\int_{\mathbb{R}^{d}} e^{i<t, x>} \mu(d t) \quad \text { and } \quad f^{*}(x)=\int_{\mathbb{R}^{d}} e^{i<t, x>} f(t) d t .
$$

For two probability measures $\mu, \nu$ on $\mathbb{R}^{d}$, we denote by $\mu \star \nu$ the convolution product of $\mu$ and $\nu$, that is the image measure of $\mu \otimes \nu$ by the application $(x, y) \rightarrow x+y$ from $\mathbb{R}^{d} \times \mathbb{R}^{d}$ to $\mathbb{R}^{d}$. If $\nu$ has a density $g$ on $\mathbb{R}^{d}$, we denote by $\mu \star g$ the density of $\mu \star \nu$, that is

$$
\mu \star g(x)=\int_{\mathbb{R}^{d}} g(x-z) \mu(d z) .
$$

\subsection{The multivariate convolution model}

Assume that one observes $n$ i.i.d. random vectors $\left(Y_{i}=\left(Y_{i, 1}, \ldots, Y_{i, d}\right)^{t}\right)_{1 \leq i \leq n}$ with values in $\mathbb{R}^{d}$ in the model

$$
Y_{i}=X_{i}+\varepsilon_{i}
$$

where the random vectors $X_{i}=\left(X_{i, 1}, \ldots, X_{i, d}\right)^{t}$ are i.i.d and distributed according to an unknown probability measure $\mu$ supported on an unknown compact subset $G$ of $\mathbb{R}^{d}$. The random vectors $\varepsilon_{i}=\left(\varepsilon_{i, 1}, \ldots \varepsilon_{i, d}\right)^{t}$ 's are also i.i.d. random and distributed according to a probability measure $\mu_{\varepsilon}$ which is supposed to be known and symmetric (that is $-\varepsilon_{1}$ has the same distribution $\mu_{\varepsilon}$ ). Hence, the distribution of the $Y_{i}^{\prime}$ 's is given by $\nu=\mu \star \mu_{\varepsilon}$.

Since $\mu_{\varepsilon}$ is symmetric, its Fourier transform $\mu_{\varepsilon}^{*}$ is a real-valued function. We also assume that

$$
\int_{\mathbb{R}^{d}}\|x\|^{6} \mu_{\varepsilon}(d x)<\infty
$$

which implies in particular that $\mu_{\varepsilon}^{*}$ is six times continuously differentiable. Finally, we assume that $\mu_{\varepsilon}^{*}$ is positive on $\mathbb{R}^{d}$.

Let $\mu_{n}$ be the empirical measure of the observations, that is

$$
\mu_{n}=\frac{1}{n} \sum_{i=1}^{n} \delta_{Y_{i}} .
$$

Under suitable assumptions, it follows from [18, that

$$
\lim _{n \rightarrow \infty} \mathbb{E}\left(W_{2}^{2}\left(\mu_{n}, \mu\right)\right)=W_{2}^{2}\left(\mu \star \mu_{\varepsilon}, \mu\right),
$$

and the term on right hand is nonzero if $\mu_{\varepsilon}$ is not the Dirac measure at 0 . Our aim is to provide an estimator $\hat{\mu}_{n}$ of the unknown distribution $\mu$ such that

$$
\lim _{n \rightarrow \infty} \mathbb{E}\left(W_{2}^{2}\left(\hat{\mu}_{n}, \mu\right)\right)=0 .
$$

\subsection{Deconvolution estimators}

Let $K$ be a symmetric density probability on $\mathbb{R}^{d}$ such that

$$
\int_{\mathbb{R}^{d}}\|x\|^{2} K(x) d x<\infty .
$$

$\mathrm{RR} \mathrm{n}^{\circ} 7678$ 
Assume moreover that its Fourier transform $K^{*}$ is compactly supported and two times differentiable with Lipschitz second derivatives. We shall give an example of such a kernel in Section 2.4.

Let $H$ be an invertible matrix from $\mathbb{R}^{d}$ to $\mathbb{R}^{d}, H^{t}$ be the transpose of $H$, and $|H|$ be the absolute value of the determinant of $H$. Define the preliminary estimator

$$
\hat{f}_{n}(x)=\frac{1}{n|H|} \sum_{i=1}^{n} \tilde{K}_{H}\left(H^{-1}\left(x-Y_{i}\right)\right)
$$

where

$$
\tilde{K}_{H}(x)=\frac{1}{(2 \pi)^{d}} \int_{\mathbb{R}^{d}} e^{i<u, x>} \frac{K^{*}(u)}{\mu_{\varepsilon}^{*}\left(\left(H^{-1}\right)^{t} u\right)} d u .
$$

The kernel $\tilde{K}_{H}$ is called the deconvolution kernel. It is well defined since $K^{*}$ is compactly supported and $\mu_{\varepsilon}^{*}$ is continuous and positive. Moreover $\tilde{K}_{H}$ belongs to $\mathbb{L}^{1}\left(\mathbb{R}^{d}\right)$ : this follows from the fact that the function $u \rightarrow K^{*}(u) / \mu_{\varepsilon}^{*}\left(\left(H^{-1}\right)^{t} u\right)$ is compactly supported and two times differentiable.

The estimator (5) is the multivariate version of the standard deconvolution kernel density estimator which was first introduced in 2 and 27. This estimator has been the subject of many works, in particular in the non-parametric univariate setting. Only few papers study the multidimensional deconvolution problem, see [6] for a recent work on this subject.

Note that $\hat{f}_{n}$ is not necessarily a density, since it has no reason to be non negative. Since our estimator has to be a probability measure, we define

$$
\hat{g}_{n}(x)=\alpha_{n} \hat{f}_{n}^{+}(x), \quad \text { where } \quad \alpha_{n}=\frac{1}{\int_{\mathbb{R}^{d}} \hat{f}_{n}^{+}(x) d x} \quad \text { and } \quad \hat{f}_{n}^{+}=\max \left\{0, \hat{f}_{n}\right\}
$$

The estimator $\hat{\mu}_{n}$ of $\mu$ is then the probability measure with density $\hat{g}_{n}$.

The first step is to prove a consistency result for this estimator, and to do this, we need to specify a loss function. The pointwise (semi-) metric and the $\mathbb{L}_{2}$ metric between probability densities are the most currently used (see for instance the monograph of [20]). Mean consistency results with respect to the $\mathbb{L}_{1}$ loss have also been proved by $[12$. However, these loss functions are not adapted to our context, since we do not assume that $\mu$ has a density.

In this paper we take $W_{2}^{2}$ as our loss function, and we give rates of convergence for the quantity $\mathbb{E}\left(W_{2}^{2}\left(\hat{\mu}_{n}, \mu\right)\right)$.

\subsection{A general decomposition}

In this section, we shall always assume that

$$
\int_{\mathbb{R}^{d}}\left(1+\|x\|^{2}\right) \sqrt{\operatorname{Var}\left(f_{n}(x)\right)} d x<\infty,
$$

which implies that $\mathbb{E}\left(W_{2}^{2}\left(\hat{\mu}_{n}, \mu\right)\right)$ is finite. More precisely, we shall prove the following "bias-variance" decomposition:

Proposition 1. Let

$$
B(H)=\int_{\mathbb{R}^{d}}\|H u\|^{2} K(u) d u \quad \text { and } \quad C(H)=B(H)+\int_{\mathbb{R}^{d}}\|x\|^{2} \mu(d x) .
$$


The following upper bound holds:

$$
\mathbb{E}\left(W_{2}^{2}\left(\hat{\mu}_{n}, \mu\right)\right) \leq 2 B(H)+4 \int_{\mathbb{R}^{d}}\left(2 C(H)+\|x\|^{2}\right) \sqrt{\operatorname{Var}\left(f_{n}(x)\right)} d x .
$$

Proof of Proposition 1. We first define the kernel $K_{H}$ by

$$
K_{H}(x)=\frac{1}{|H|} K\left(H^{-1} x\right) .
$$

As usual in deconvolution problems, the estimator $\hat{f}_{n}$ is build in such a way that $\mathbb{E}\left(\hat{f}_{n}(x)\right)=\mu \star K_{H}(x)$. Indeed, by Plancherel's identity

$$
\begin{aligned}
\mathbb{E}\left(\hat{f}_{n}(x)\right) & =\frac{1}{|H|} \int_{\mathbb{R}^{d}} \tilde{K}_{H}\left(H^{-1}(x-z)\right) \mu \star \mu_{\varepsilon}(z) d z \\
& =\frac{1}{(2 \pi)^{d}} \int_{\mathbb{R}^{d}}\left(\frac{1}{|H|} \tilde{K}_{H}\left(H^{-1}(x-\cdot)\right)^{*}(u) \mu^{*}(u) \mu_{\varepsilon}^{*}(u) d u\right.
\end{aligned}
$$

Since $K_{H}$ is symmetric, we have that

$$
\left(\frac{1}{|H|} \tilde{K}_{H}\left(H^{-1}(x-\cdot)\right)^{*}(u)=e^{i<u, x>} \tilde{K}_{H}^{*}\left(-H^{t} u\right)=e^{i<u, x>} \tilde{K}_{H}^{*}\left(H^{t} u\right),\right.
$$

and by definition of $\tilde{K}_{H}$,

$$
\begin{aligned}
\mathbb{E}\left(\hat{f}_{n}(x)\right) & =\frac{1}{(2 \pi)^{d}} \int_{\mathbb{R}^{d}} e^{i<u, x>} \frac{K^{*}\left(H^{t} u\right)}{\mu_{\varepsilon}^{*}(u)} \mu^{*}(u) \mu_{\varepsilon}^{*}(u) d u \\
& =\frac{1}{(2 \pi)^{d}} \int_{\mathbb{R}^{d}}\left(\frac{1}{|H|} K\left(H^{-1}(x-\cdot)\right)^{*}(u) \mu^{*}(u) d u=\mu \star K_{H}(x) .\right.
\end{aligned}
$$

Now, by the triangle inequality

$$
W_{2}^{2}\left(\hat{\mu}_{n}, \mu\right) \leq 2 W_{2}^{2}\left(\mu \star K_{H}, \mu\right)+2 W_{2}^{2}\left(\hat{\mu}_{n}, \mu \star K_{H}\right) .
$$

The first term on the right hand side of (7) is deterministic, and can be easily bounded as follows: let $Y_{H}$ be a random variable with distribution $K_{H}$ and independent of $X_{1}$, in such a way that the distribution of $X_{1}+Y_{H}$ is $\mu \star K_{H}$. By definition of $W_{2}$, one has

$$
W_{2}^{2}\left(\mu \star K_{H}, \mu\right) \leq \mathbb{E}\left(\left\|X_{1}+Y_{H}-X_{1}\right\|^{2}\right)=\mathbb{E}\left(\left\|Y_{H}\right\|_{2}^{2}\right)=B(H)
$$

To control the second term of (7), we shall use the following lemma

Lemma 1. Let $\mu$ and $\nu$ be two probability measures on $\mathbb{R}^{d}$, and let $|\mu-\nu|$ be the total variation measure of $\mu-\nu$. Then

$$
W_{2}^{2}(\mu, \nu) \leq 2 \min _{a \in \mathbb{R}^{d}} \int_{\mathbb{R}^{d}}\|x-a\|^{2}|\mu-\nu|(d x) .
$$

In particular, if $\mu$ and $\nu$ have respective densities $f$ and $g$ with respect to the Lebesgue measure

$$
W_{2}^{2}(\mu, \nu) \leq 2 \min _{a \in \mathbb{R}^{d}} \int_{\mathbb{R}^{d}}\|x-a\|^{2}|f(x)-g(x)| d x .
$$


Remark 1. The inequality (9) has been proved by [29] with the constant 4, and by [18] with the constant 3. We give here a very elementary proof which provides a better constant.

Remark 2. If $\mu$ has a density $f_{\mu}$ with respect to the Lebesgue measure on $\mathbb{R}^{d}$, we can use the inequality (9) to obtain the following upper bound for the first term of (7)

$$
W_{2}^{2}\left(\mu \star K_{H}, \mu\right) \leq 2 \int_{\mathbb{R}^{d}}\|x\|^{2}\left|f_{\mu}(x)-\mu \star K_{H}(x)\right| d x .
$$

Now, as in density deconvolution, if $f_{\mu}$ is smooth enough, this upper bound may be more precise than the simple upper bound $W_{2}^{2}\left(\mu \star K_{H}, \mu\right) \leq B(H)$. However, the fact that $f_{\mu}$ exists and is smooth on $\mathbb{R}^{d}$ is a very restrictive assumption in our context. Indeed, the basic case that we want to recover is that where $\mu$ is uniformly distributed on the compact set $G$. In that case, the density $f_{\mu}$ may not exist at all, and if it exists, it is not regular at the boundary of $G$, so that the upper bound $W_{2}^{2}\left(\mu \star K_{H}, \mu\right) \leq B(H)$ is always better than (10). Note also that the upper bound $W_{2}^{2}\left(\mu \star K_{H}, \mu\right) \leq B(H)$ is in fact an equality if $\mu$ is a Dirac measure, and hence it cannot be improved without additional assumptions on $\mu$.

Proof of Lemma 1. Let $\mu-\nu=\pi_{+}-\pi_{-}$be the Hahn-Jordan decomposition of $\mu-\nu$. From the proof of Theorem 2.6.1 of [26, we know that

$$
W_{2}^{2}(\mu, \nu)=W_{2}^{2}\left(\pi_{+}, \pi_{-}\right) .
$$

By the triangle inequality, for any $a \in \mathbb{R}^{d}$,

$$
W_{2}^{2}\left(\pi_{+}, \pi_{-}\right) \leq 2 W_{2}^{2}\left(\pi_{+}\left(\mathbb{R}^{d}\right) \delta_{a}, \pi_{+}\right)+2 W_{2}^{2}\left(\pi_{-}\left(\mathbb{R}^{d}\right) \delta_{a}, \pi_{-}\right) .
$$

Now

$$
W_{2}^{2}\left(\pi_{+}\left(\mathbb{R}^{d}\right) \delta_{a}, \pi_{+}\right)=\int_{\mathbb{R}^{d}}\|x-a\|^{2} \pi_{+}(d x)
$$

and

$$
W_{2}^{2}\left(\pi_{-}\left(\mathbb{R}^{d}\right) \delta_{a}, \pi_{-}\right)=\int_{\mathbb{R}^{d}}\|x-a\|^{2} \pi_{-}(d x) .
$$

Finally,

$$
W_{2}^{2}(\mu, \nu)=W_{2}^{2}\left(\pi_{+}, \pi_{-}\right) \leq 2 \int\|x-a\|^{2}\left(\pi_{+}+\pi_{-}\right)(d x)
$$

and the result follows.

We continue the proof of Proposition 1. Applying Lemma 1 we have successively

$$
\begin{aligned}
& W_{2}^{2}\left(\hat{\mu}_{n}, \mu \star K_{H}\right) \leq 2 \int_{\mathbb{R}^{d}}\|x\|^{2}\left|\alpha_{n} \hat{f}_{n}^{+}(x)-\mathbb{E}\left(\hat{f}_{n}(x)\right)\right| d x \\
\leq & 2 \alpha_{n} \int_{\mathbb{R}^{d}}\|x\|^{2}\left|\hat{f}_{n}^{+}(x)-\mathbb{E}\left(\hat{f}_{n}(x)\right)\right| d x+2\left(1-\alpha_{n}\right) \int_{\mathbb{R}^{d}}\|x\|^{2} \mathbb{E}\left(\hat{f}_{n}(x)\right) d x \\
\leq & 2 \int_{\mathbb{R}^{d}}\|x\|^{2}\left|f_{n}(x)-\mathbb{E}\left(\hat{f}_{n}(x)\right)\right|+2\left(1-\alpha_{n}\right) \int_{\mathbb{R}^{d}}\|x\|^{2} \mathbb{E}\left(\hat{f}_{n}(x)\right) d x
\end{aligned}
$$


Note that

$$
\int_{\mathbb{R}^{d}}\|x\|^{2} \mathbb{E}\left(\hat{f}_{n}(x)\right) d x \leq 2 C(H) \quad \text { and } \quad\left(1-\alpha_{n}\right) \leq \int_{\mathbb{R}^{d}}\left(\hat{f}_{n}^{+}(x)-\hat{f}_{n}(x)\right) d x,
$$

and consequently

$$
\begin{aligned}
\mathbb{E}\left(\left(1-\alpha_{n}\right) \int_{\mathbb{R}^{d}}\|x\|^{2} \mathbb{E}\left(\hat{f}_{n}(x)\right) d x\right) & \leq 2 C(H) \mathbb{E}\left(\int_{\mathbb{R}^{d}}\left(\hat{f}_{n}^{+}(x)-\mathbb{E}\left(\hat{f}_{n}(x)\right)\right) d x\right) \\
& \leq 2 C(H) \mathbb{E}\left(\int_{\mathbb{R}^{d}}\left|\hat{f}_{n}(x)-\mathbb{E}\left(\hat{f}_{n}(x)\right)\right| d x\right) .(12)
\end{aligned}
$$

Since $\mathbb{E}\left(\left|\hat{f}_{n}(x)-\mathbb{E}\left(\hat{f}_{n}(x)\right)\right|\right) \leq\left(\operatorname{Var}\left(\hat{f}_{n}(x)\right)\right)^{1 / 2}$, Proposition 1 follows from (7), (8), (11) and (12).

\subsection{Errors with independent coordinates}

In this section, we assume that the random variables $\left(\varepsilon_{1, j}\right)_{1 \leq j \leq d}$ are independent, which means that $\varepsilon_{1}$ has the distribution $\mu_{\varepsilon}=\mu_{1} \otimes \mu_{2} \otimes \cdots \otimes \mu_{d}$.

In this context, we shall use the kernel

$$
K=k^{\otimes n}, \quad \text { where } \quad k(x)=\frac{3}{8 \pi}\left(\frac{4 \sin (x / 4)}{x}\right)^{4} .
$$

Note that $k^{*}(x)=3 g(4|t|) / 16$, with

$$
g(t)=\left(\frac{t^{3}}{2}-2 t^{2}+\frac{16}{3}\right) \mathbf{1}_{[0,2[}(t)+\left(\frac{-t^{3}}{6}+2 t^{2}-8 t+\frac{32}{3}\right) \mathbf{1}_{[2,4[}(t) .
$$

The kernel $K$ is a symmetric density, and $K^{*}$ is supported over $[-1,1]^{d}$. Moreover, since $t \rightarrow g(|t|)$ is two times differentiable with Lipschitz second derivative, the kernel $K$ satisfies all the required conditions.

We choose a diagonal matrix $H$ with positive diagonal terms $h_{1}, h_{2}, \ldots, h_{d}$. The kernel $\tilde{K}_{H}$ defined in $(6)$ is given by

$$
\tilde{K}_{H}=\tilde{k}_{1, h_{1}} \otimes \tilde{k}_{2, h_{2}} \otimes \cdots \otimes \tilde{k}_{d, h_{d}} \quad \text { where } \quad \tilde{k}_{j, h_{j}}(x)=\frac{1}{2 \pi} \int e^{i u x} \frac{k^{*}(u)}{\mu_{j}^{*}\left(u / h_{j}\right)} d u \text {. }
$$

The preliminary estimator $\hat{f}_{n}$ defined in 5 is then

$$
\hat{f}_{n}\left(x_{1}, \ldots, x_{d}\right)=\frac{1}{n} \sum_{i=1}^{n} \prod_{j=1 \ldots d} \frac{1}{h} \tilde{k}_{j} h_{j}\left(\frac{x_{j}-Y_{i, j}}{h_{j}}\right)
$$

and the estimator $\hat{\mu}_{n}$ of $\mu$ is deduced from $\hat{f}_{n}$ as in Section 2.2

Note that $B(H)=\beta\left(h_{1}^{2}+\cdots+h_{d}^{2}\right)$, with $\beta=\int u^{2} k(u) d u$. To ensure the consistency of the estimator, the bias term $B(H)$ has to tend to zero as $n$ tends to infinity. Without loss of generality, we assume in the following that $H$ is such that $B(H) \leq 1$. Hence, the variance term

$$
V_{n}=4 \int_{\mathbb{R}^{d}}\left(2 C(H)+\|x\|^{2}\right) \sqrt{\operatorname{Var}\left(\hat{f}_{n}(x)\right)} d x
$$


in Proposition 1 is such that

$$
V_{n} \leq C \int_{\mathbb{R}^{d}}\left(1+\sum_{i=1}^{d} x_{i}^{2}\right) \sqrt{\operatorname{Var}\left(\hat{f}_{n}\left(x_{1}, \ldots, x_{n}\right)\right)} d x_{1} \ldots d x_{d}
$$

for some positive constant $C$ that only depends on $\mu$ via the quantity $M=$ $\sup _{1 \leq i \leq d}\left|X_{1, i}\right|_{\infty}$ where $|\cdot|_{\infty}$ is the essential-supremum norm. Now

$$
\sqrt{\operatorname{Var}\left(\hat{f}_{n}\left(x_{1}, \ldots, x_{n}\right)\right)} \leq \frac{1}{\sqrt{n}} \sqrt{\mathbb{E}\left(\left(\prod_{i=1}^{d} \frac{1}{h_{i}} \tilde{k}_{i, h_{i}}\left(\frac{x_{i}-Y_{1, i}}{h_{i}}\right)\right)^{2}\right)} .
$$

Applying Cauchy-Schwarz's inequality $d$-times, we obtain that

$$
\begin{aligned}
& \int_{\mathbb{R}^{d}} \sqrt{\operatorname{Var}\left(\hat{f}_{n}\left(x_{1}, \ldots, x_{n}\right)\right)} d x_{1} \ldots d x_{d} \\
\leq & \frac{D_{1}}{\sqrt{n}} \sqrt{\mathbb{E}\left(\prod_{i=1}^{d} \int\left(1 \vee x_{i}^{2}\right)\left(\frac{1}{h_{i}} \tilde{k}_{i, h_{i}}\left(\frac{x_{i}-Y_{1, i}}{h_{i}}\right)\right)^{2} d x_{i}\right)} \\
\leq & \frac{D_{2}}{\sqrt{n}} \sqrt{\mathbb{E}\left(\prod_{i=1}^{d}\left(1 \vee Y_{1, i}^{2}\right) \int\left(1+u_{i}^{2} h_{i}^{2}\right) \frac{1}{h_{i}}\left(\tilde{k}_{i, h_{i}}(u)\right)^{2} d u_{i}\right)}
\end{aligned}
$$

where $D_{1}$ and $D_{2}$ are positive constants depending on $d$. Now, $Y_{1, i}^{2} \leq 2\left(M^{2}+\right.$ $\left.\varepsilon_{1, i}^{2}\right)$ and using the independence of the coordinates of $\varepsilon_{1}$, we obtain that

$\int_{\mathbb{R}^{d}} \sqrt{\operatorname{Var}\left(\hat{f}_{n}(x)\right)} d x \leq \frac{D_{3}}{\sqrt{n}} \sqrt{\left(\prod_{i=1}^{d}\left(M^{2}+\mathbb{E}\left(\varepsilon_{1, i}^{2}\right)\right) \int\left(1+u_{i}^{2} h_{i}^{2}\right) \frac{1}{h_{i}}\left(\tilde{k}_{i, h_{i}}(u)\right)^{2} d u_{i}\right)}$,

It follows that

$$
\int_{\mathbb{R}^{d}} \sqrt{\operatorname{Var}\left(\hat{f}_{n}(x)\right)} d x \leq \frac{A_{0}}{\sqrt{n}} \sqrt{\prod_{i=1}^{d} \int\left(1+u_{i}^{2} h_{i}^{2}\right) \frac{1}{h_{i}}\left(\tilde{k}_{i, h_{i}}(u)\right)^{2} d u_{i}} .
$$

In the same way, we have that

$$
\begin{aligned}
\int_{\mathbb{R}^{d}} x_{k}^{2} \sqrt{\operatorname{Var}\left(\hat{f}_{n}(x)\right)} d x & \leq \frac{A_{k}}{\sqrt{n}} \sqrt{\left(M^{6}+\mathbb{E}\left(\varepsilon_{1, i}^{6}\right)\right) \int\left(1+u_{k}^{6} h_{k}^{6}\right) \frac{1}{h_{k}}\left(\tilde{k}_{k, h_{k}}(u)\right)^{2} d u_{k}} \\
& \times \sqrt{\prod_{i \neq k}\left(M+\mathbb{E}\left(\varepsilon_{1, i}^{2}\right)\right) \int\left(1+u_{i}^{2} h_{i}^{2}\right) \frac{1}{h_{i}}\left(\tilde{k}_{i, h_{i}}(u)\right)^{2} d u_{i}} .
\end{aligned}
$$

Note that $\mathbb{E}\left(\varepsilon_{1, i}^{2}\right)$ and $\mathbb{E}\left(\varepsilon_{1, i}^{6}\right)$ are finished according to (3). Starting from these computations, one can prove the following Proposition.

Proposition 2. Let $r_{i}(x)=1 / \mu_{i}^{*}(x)$, and let $\left(h_{1}, \ldots, h_{d}\right) \in[0,1]^{d}$. The following upper bound holds

$\mathbb{E}\left(W_{2}^{2}\left(\hat{\mu}_{n}, \mu\right)\right) \leq 2 \beta\left(h_{1}^{2}+\cdots+h_{d}^{2}\right)+\frac{L}{\sqrt{n}}\left(\prod_{i=1}^{d} I_{i}\left(h_{i}\right)+\sum_{k=1}^{d} J_{k}\left(h_{k}\right)\left(\prod_{i=1, i \neq k}^{d} I_{i}\left(h_{i}\right)\right)\right)$

$\mathrm{RR} \mathrm{n}^{\circ} 7678$ 
where $L$ is some positive constant depending on $d, M$ and $\left(\mathbb{E}\left(\varepsilon_{1, i}^{2}\right), \mathbb{E}\left(\varepsilon_{1, i}^{6}\right)\right)_{1 \leq i \leq d}$, and

$$
\begin{aligned}
I_{i}(h) \leq & \sqrt{\int_{-1 / h}^{1 / h}\left(r_{i}(u)\right)^{2}+\left(r_{i}^{\prime}(u)\right)^{2} d u} \\
J_{i}(h) \leq & \sqrt{\int_{-1 / h}^{1 / h}\left(r_{i}(u)\right)^{2}+\left(r_{i}^{\prime \prime \prime}(u)\right)^{2} d u} \\
& +h \sqrt{\int_{-1 / h}^{1 / h}\left(r_{i}^{\prime \prime}(u)\right)^{2} d u}+h^{2} \sqrt{\int_{-1 / h}^{1 / h}\left(r_{i}^{\prime}(u)\right)^{2} d u} .
\end{aligned}
$$

Remark 3. Note that the upper bound in Proposition 2 depends on the unknown distribution $\mu$ only through the constant $M$ (which appears in (15) and (17)). Hence the rate of convergence of $\hat{\mu}$ obtained from Proposition 2 does not depend on $\mu$. Note that in the classical context of $\mathbb{L}_{2}$-density deconvolution, the variance term is exactly

$$
\int_{\mathbb{R}^{d}} \operatorname{Var}\left(\hat{f}_{n}(x)\right) d x
$$

which can be bounded independently of $\mu$. This leads to the idea that $\mathbb{E}\left(W_{2}^{2}\left(\hat{\mu}_{n}, \mu\right)\right)$ depends very poorly of the unknown distribution $\mu$. If this intuition is correct, a possible way to select the bandwidth parameter $\mathbf{h}=\left(h_{1}, \ldots, h_{d}\right)$ is to choose by simulation the best possible $\mathbf{h}$ in the simple case $\mu=\delta_{0}$. This will be done in Section 3. We shall see that this selected $\mathbf{h}$ leads to very good results for different choices of $\mu$, even when $\mu$ has a density (see Section 3.2).

Proof of Proposition 2, By Plancherel's identity,

$$
\begin{aligned}
\int \frac{1}{h}\left(\tilde{k}_{i, h}(u)\right)^{2} d u=\frac{1}{2 \pi} \int \frac{1}{h} \frac{\left(k^{*}(u)\right)^{2}}{\left(\mu_{i}^{*}(u / h)\right)^{2}} d u & =\frac{1}{2 \pi} \int \frac{\left(k^{*}(h u)\right)^{2}}{\left(\mu_{i}^{*}(u)\right)^{2}} d u \\
& \leq \frac{1}{2 \pi} \int_{-1 / h}^{1 / h} r_{i}^{2}(u) d u
\end{aligned}
$$

the last upper bound being true because $k^{*}$ is supported over $[-1,1]$ and bounded by 1 .

Let $C$ be a positive constant, which may vary from line to line. Let $q_{i, h}(u)=$ $r_{i}(u / h) k^{*}(u)$. Since $q_{i, h}$ is differentiable with compactly supported derivative, we have that

$$
-i u \tilde{k}_{i, h}(u)=\left(q_{i, h}^{\prime}\right)^{*}(u) .
$$

Applying Plancherel's identity again,

$$
\begin{aligned}
\int h u^{2}\left(\tilde{k}_{i, h}(u)\right)^{2} d u & =\frac{1}{2 \pi} \int h\left(q_{i, h}^{\prime}(u)\right)^{2} d u \\
\leq C & \left(\int_{-1 / h}^{1 / h}\left(r_{i}^{\prime}(u)\right)^{2} d u+h^{2} \int_{-1 / h}^{1 / h} r_{i}^{2}(u) d u\right),
\end{aligned}
$$

the last inequality being true because $k^{*}$ and $\left(k^{*}\right)^{\prime}$ are compactly supported over $[-1,1]$. Consequently

$$
\sqrt{\int\left(1+u_{i}^{2} h_{i}^{2}\right) \frac{1}{h_{i}}\left(\tilde{k}_{i, h_{i}}(u)\right)^{2} d u_{i}} \leq C I_{i}\left(h_{i}\right) .
$$

$\mathrm{RR} \mathrm{n}^{\circ} 7678$ 
In the same way

$$
-i u^{3} \tilde{k}_{i, h}(u)=\left(q_{i, h}^{\prime \prime \prime}\right)^{*}(u) \text { and } \int h^{5} u^{6}\left(\tilde{k}_{i, h}(u)\right)^{2} d u=\frac{1}{2 \pi} \int h^{5}\left(q_{i, h}^{\prime \prime \prime}(u)\right)^{2} d u,
$$

Now, since $k^{*},\left(k^{*}\right)^{\prime},\left(k^{*}\right)^{\prime \prime}$ and $\left(k^{*}\right)^{\prime \prime \prime}$ are compactly supported over $[-1,1]$,

$$
\begin{aligned}
\int h^{5}\left(q_{i, h}^{\prime \prime \prime}(u)\right)^{2} d u \leq & C\left(\int_{-1 / h}^{1 / h}\left(r_{i}^{\prime \prime \prime}(u)\right)^{2} d u+h^{2} \int_{-1 / h}^{1 / h}\left(r_{i}^{\prime \prime}(u)\right)^{2} d u\right. \\
& \left.+h^{4} \int_{-1 / h}^{1 / h}\left(r_{i}^{\prime}(u)\right)^{2} d u+h^{6} \int_{-1 / h}^{1 / h}\left(r_{i}(u)\right)^{2} d u\right) .
\end{aligned}
$$

Consequently

$$
\sqrt{\int\left(1+u_{k}^{6} h_{k}^{6}\right) \frac{1}{h_{k}}\left(\tilde{k}_{k, h_{k}}(u)\right)^{2} d u_{k}} \leq C J_{k}\left(h_{k}\right) .
$$

The results follows.

\subsection{Linear transform of errors with independent coordi- nates}

In this section, we assume that $\varepsilon=A \eta$, where the distribution $\mu_{\eta}$ of $\eta$ is such that $\mu_{\eta}=\mu_{1} \otimes \mu_{2} \otimes \cdots \otimes \mu_{d}$, and $A$ is some known invertible matrix. Applying $A^{-1}$ to the random variables $Y_{i}$ in $(2)$, we obtain the new model

$$
A^{-1} Y_{i}=A^{-1} X_{i}+\eta_{i}
$$

that is: a convolution model in which the error has independent coordinates.

To estimate the image measure $\mu^{A^{-1}}$ of $\mu$ by $A^{-1}$, we use the preliminary estimator of Section 2.4 that is

$$
\hat{f}_{n, A^{-1}}\left(x_{1}, \ldots, x_{d}\right)=\frac{1}{n} \sum_{i=1}^{n} \prod_{j=1 \ldots d} \frac{1}{h} \tilde{k}_{j, h_{j}}\left(\frac{x_{j}-\left(A^{-1} Y_{i}\right)_{j}}{h_{j}}\right),
$$

and the estimator $\hat{\mu}_{n, A^{-1}}$ of $\mu^{A^{-1}}$ is deduced from $\hat{f}_{n, A^{-1}}$ as in Section 2.2. This estimator $\hat{\mu}_{n, A^{-1}}$ has the density $\hat{g}_{n, A^{-1}}$ with respect to the Lebesgue measure.

To estimate $\mu$, we define $\hat{\mu}_{n}=\hat{\mu}_{n, A^{-1}}^{A}$ as the image measure of $\hat{\mu}_{n, A^{-1}}$ by $A$. This estimator has the density $\hat{g}_{n}=|A|^{-1} \hat{g}_{n, A^{-1}} \circ A^{-1}$ with respect to the Lebesgue measure. It can be deduced from the preliminary estimator $\hat{f}_{n}=|A|^{-1} \hat{f}_{n, A^{-1}} \circ A^{-1}$ as in Section 2.2. Now

$$
\begin{aligned}
W_{2}^{2}\left(\hat{\mu}_{n}, \mu\right) & =\min _{\lambda \in \Pi\left(\hat{\mu}_{n}, \mu\right)} \int\|x-y\|^{2} \lambda(d x, d y) \\
& =\min _{\pi \in \Pi\left(\hat{\mu}_{\left.n, A^{-1}, \mu^{A^{-1}}\right)} \int\|A(x-y)\|^{2} \pi(d x, d y) .\right.} .
\end{aligned}
$$

Consequently, if $\|A\|=\sup _{\|x\|=1}\|A x\|$, we obtain that

$$
W_{2}^{2}\left(\hat{\mu}_{n}, \mu\right) \leq\|A\| W_{2}^{2}\left(\hat{\mu}_{n, A^{-1}}, \mu^{A^{-1}}\right),
$$

which is an equality if $A$ is an unitary matrix. Hence the upper bound given in Proposition 2 for the quantity $\mathbb{E}\left(W_{2}^{2}\left(\hat{\mu}_{n, A^{-1}}, \mu^{A^{-1}}\right)\right)$ is also valid for $\mathbb{E}\left(W_{2}^{2}\left(\hat{\mu}_{n}, \mu\right)\right)$.

Note that $\hat{f}_{n}$ can be written as in $(5)$, with the kernel $K=|A|^{-1} k^{\otimes n} \circ A^{-1}$ and the diagonal matrix $H$ with diagonal terms $h_{1}, \ldots, h_{d}$. 


\subsection{Examples of rates of convergence}

In this section we shall always assume that $\mu_{\varepsilon}=\mu_{1} \otimes \mu_{2} \otimes \cdots \otimes \mu_{d}$. According to the comments of Section 2.5, the rates of convergence are also valid for any linear invertible transform of such noises.

Case 1: no noise. In that case $\mu_{1}^{*}=\mu_{2}^{*}=\ldots=\mu_{d}^{*}=1$. Taking $h_{1}=h_{2}=$ $\cdots=h_{d}=h$, Proposition 2 gives the upper bound

$$
\mathbb{E}\left(W_{2}^{2}\left(\hat{\mu}_{n}, \mu\right)\right) \leq C\left(h^{2}+\frac{1}{\sqrt{n h^{d}}}\right) .
$$

Taking $h=n^{-1 /(d+4)}$, we obtain the rate of convergence

$$
\mathbb{E}\left(W_{2}^{2}\left(\hat{\mu}_{n}, \mu\right)\right) \leq \frac{C}{n^{2 /(d+4)}} .
$$

Note that this is the same rate as that obtained by [18] for the empirical measure $\mu_{n}$ defined in (4). To our knowledge, this rate of convergence has not been improved without making additional assumptions on $\mu$. 225] proved some upper and lower bounds (Theorem 11.1.6) for $\mathbb{E}\left(W_{2}^{2}\left(\mu_{n}, \mu\right)\right)$ under entropy conditions on $\mu$. It follows from his estimates that if $\mu$ has a density and $d$ is even, then the rate $\mathbb{E}\left(W_{2}^{2}\left(\mu_{n}, \mu\right)\right) \leq C n^{-2 / d}$ is optimal.

Case 2: convolution of Laplace noise. We consider the case where

$$
\mu_{i}^{*}(u)=\frac{1}{\left(1+u^{2}\right)^{k_{i}}},
$$

$k_{i}$ being nonnegative integers. This corresponds to the case where the density of $\varepsilon_{1, i}$ is the $k_{i}$-times convolution of the Laplace density.

Proposition 2 gives the upper bound

$$
\mathbb{E}\left(W_{2}^{2}\left(\hat{\mu}_{n}, \mu\right)\right) \leq C\left(h_{1}^{2}+\cdots+h_{d}^{2}+\frac{1}{\sqrt{n}} \prod_{i=1}^{d} h_{i}^{-\left(4 k_{i}+1\right) / 2}\right) .
$$

This bound is similar to the $\mathbb{L}_{2}$-risk bound obtained by [6] in the context of multivariate density deconvolution with an ordinary smooth noise (see Section 3.2.1 in their paper). Their computations show that one can take $h_{i}=$ $n^{-1 /\left(d+4\left(1+k_{1}+\cdots+k_{d}\right)\right)}$ and then obtain the rate of convergence

$$
\mathbb{E}\left(W_{2}^{2}\left(\hat{\mu}_{n}, \mu\right)\right) \leq \frac{C}{n^{2 /\left(d+4\left(1+k_{1}+\cdots+k_{d}\right)\right)}}
$$

In particular:

- If $k_{i}=0$ for all $i$ (no noise), we obtain the same rate as previously.

- If $k_{i}=1$ for all $i$ (isotropic Laplace noise), we obtain the rate

$$
\mathbb{E}\left(W_{2}^{2}\left(\hat{\mu}_{n}, \mu\right)\right) \leq \frac{C}{n^{2 /(5 d+4)}} .
$$

- If $k_{\ell}=1$ and $k_{i}=0$ for $i \neq \ell$ (Laplace noise in one direction), we obtain the rate

$$
\mathbb{E}\left(W_{2}^{2}\left(\hat{\mu}_{n}, \mu\right)\right) \leq \frac{C}{n^{2 /(d+8)}} .
$$


The index $I(d)=k_{1}+\cdots+k_{d}$ can be seen as an index of global regularity of the error distribution, summing all the regularities $k_{i}$ of the marginal distributions. As usual in deconvolution problems, the worst rates of convergence are obtained for very regular error distributions: more precisely, the rate of convergence becomes slower as $I(d)$ increases. Note also that a single marginal distribution with regularity $N$ gives the same rates as $N$ marginal distributions with regularity 1.

Case 3: isotropic Gaussian noise. We consider the case where

$$
\mu_{1}^{*}(u)=\mu_{2}^{*}(u)=\ldots=\mu_{d}^{*}(u)=\exp \left(-u^{2} / 2\right) .
$$

Proposition 2 gives the upper bound

$$
\mathbb{E}\left(W_{2}^{2}\left(\hat{\mu}_{n}, \mu\right)\right) \leq C\left(h_{1}^{2}+\cdots+h_{d}^{2}+\frac{1}{\sqrt{n}} \prod_{i=1}^{d} h_{i}^{-5 / 2} \exp \left(h_{i}^{-2} / 2\right)\right) .
$$

Following again [6], one can take $h_{i}=\sqrt{2 / \log (n)}$, and we obtain the rate of convergence

$$
\mathbb{E}\left(W_{2}^{2}\left(\hat{\mu}_{n}, \mu\right)\right) \leq \frac{C}{\log (n)} .
$$

Case 4: Gaussian noise in one direction. We consider the case where $\mu_{1}^{*}(u)=\exp \left(-u^{2} / 2\right)$, and $\mu_{2}^{*}=\cdots=\mu_{d}^{*}=1$. Taking $h_{2}=h_{3}=\cdots=h_{d}=h$, Proposition 2 gives the upper bound

$$
\mathbb{E}\left(W_{2}^{2}\left(\hat{\mu}_{n}, \mu\right)\right) \leq C\left(h_{1}^{2}+h^{2}+\frac{1}{\sqrt{n h^{d-1} h_{1}^{5}}} \exp \left(h_{1}^{-2} / 2\right)\right) .
$$

Taking $h_{1}=\sqrt{2 / \log (n)}$ and $h=n^{-1 /(5 d-5)}$, we obtain the rate of convergence

$$
\mathbb{E}\left(W_{2}^{2}\left(\hat{\mu}_{n}, \mu\right)\right) \leq \frac{C}{\log (n)} .
$$

Hence, a Gaussian noise in one single direction gives the same rate of convergence as an isotropic Gaussian noise. This is coherent with the discussion in Section 3.2 .2 of [6] about density deconvolution in $\mathbb{R}^{d}$.

\section{$3 \quad$ Experiments}

In this section, we take $d=2$ and we consider the case where $\mu_{\varepsilon}=\mu_{1} \otimes \mu_{2}$. For all the following experiments the preliminary estimator $\hat{f}_{n}$ is defined as in 14 with the bandwidth parameter $\mathbf{h}=\left(h_{1}, h_{2}\right)$ and the kernel

$$
K=k^{\otimes n}, \quad \text { where } \quad k(x)=\frac{3}{16 \pi}\left(\frac{8 \sin (x / 8)}{x}\right)^{4} .
$$

The only difference with the kernel given in (13) is that $K$ is now supported over $[-1 / 2,1 / 2]^{d}$. The estimator $\hat{\mu}_{n}$ of $\mu$ is then deduced from $\hat{f}_{n}$ as in Section 2.2 
In practice, the deconvolution estimator $\hat{\mu}_{n}=\hat{\mu}_{n, \mathbf{h}}$ is only computed on a finite set of locations. Let $\mathcal{P}=\{p\}$ be a finite regular grid of points in $\mathbb{R}^{2}$, a discrete version $\tilde{\mu}_{n}=\tilde{\mu}_{n, \mathbf{h}}$ of $\hat{\mu}_{n, \mathbf{h}}$ is defined by

$$
\tilde{\mu}_{n, \mathbf{h}}=\sum_{p \in \mathcal{P}} \hat{\alpha}_{p}(\mathbf{h}) \delta_{p}
$$

where

$$
\hat{\alpha}_{p}(\mathbf{h})=\frac{\hat{f}_{n}^{+}(p)}{\sum_{p \in \mathcal{P}} \hat{f}_{n}^{+}(p)} .
$$

Note that the $W_{2}$ distance between $\tilde{\mu}_{n, \mathbf{h}}$ and $\hat{\mu}_{n, \mathbf{h}}$ tends to zero as the grid resolution tends to zero. In the following, it is assumed that the grid resolution is chosen small enough, namely it is assumed that

$$
W_{2}^{2}\left(\tilde{\mu}_{n}, \hat{\mu}_{n}\right) \ll W_{2}^{2}\left(\mu, \hat{\mu}_{n}\right) .
$$

\subsection{Dirac experiment and bandwidth selection.}

One situation for which the Wasserstein distance $W_{2}$ is computable is the case where $\mu$ is a Dirac measure. Obviously this framework has no interest in practice but it allows us to validate the results proved in Section 2. As we shall see, it is also a way to select a bandwidth which will be a reasonable candidate in a general context.

Let $\mu=\delta_{0}$, which corresponds to the case where $Y_{i}=\varepsilon_{i}$ in the convolution model (2). Assume that $\mu_{1}^{*}(u)=\mu_{2}^{*}(u)=\left(1+u^{2}\right)^{-1}$, which means that $\varepsilon_{1,1}$ and $\varepsilon_{1,2}$ have a standard Laplace distribution with variance 2 . For this Laplace isotropic noise, we choose $\mathbf{h}=(h, h)$.

For the empirical measure $\mu_{n}$ defined in (4), one has

$$
\mathbb{E}\left(W_{2}^{2}\left(\mu_{n}, \delta_{0}\right)\right)=\mathbb{E}\left(\int_{\mathbb{R}^{2}}\|x\|^{2} \mu_{n}(x) d x\right)=\operatorname{Var}\left(\varepsilon_{1,1}\right)+\operatorname{Var}\left(\varepsilon_{1,2}\right)=4
$$

For $\tilde{\mu}_{n}$, one has

$$
\mathbb{E}\left(W_{2}^{2}\left(\tilde{\mu}_{n}, \delta_{0}\right)\right)=\mathbb{E}\left(\sum_{p \in \mathcal{P}}\|p\|^{2} \hat{\alpha}_{p}(\mathbf{h})\right) .
$$

Let $I_{n}(h)=W_{2}^{2}\left(\tilde{\mu}_{n}, \delta_{0}\right)$ be the Wasserstein distance between $\delta_{0}$ and $\tilde{\mu}_{n}$. For a given $h$ in a grid $\mathcal{H}$ of possible bandwidths, $\mathbb{E}\left(I_{n}(h)\right)$ can be approximated with an elementary Monte Carlo method by repeating the simulation $N_{s}$ times. Figure 1 shows the boxplot of the distribution of $I_{n}(h)$ on a rough grid of bandwidth with $n=20000$. For such a sample size, the deconvolution estimator $\tilde{\mu}_{n}$ performs better than the empirical measure on a large scale of bandwidth values.

For each $n$, an approximation of $h_{*}=\operatorname{argmin} \mathbb{E}\left(W^{2}\left(\hat{\mu}_{n, h}, \delta_{0}\right)\right)$ can be computed as follows

$$
\hat{h}_{*}(n)=\operatorname{argmin}_{h \in \mathcal{H}} \bar{I}_{n}(h) \quad \text { where } \quad \bar{I}_{n}(h)=\frac{1}{N_{s}} \sum_{s=1}^{N_{s}} I_{n, s}(h) .
$$

and $I_{n, s}(h)$ is the computation of $I_{n}(h)$ corresponding to the $s$-th simulation. Table 1 gives the value of $\hat{h}_{*}(n)$ computed for different sample sizes and the 


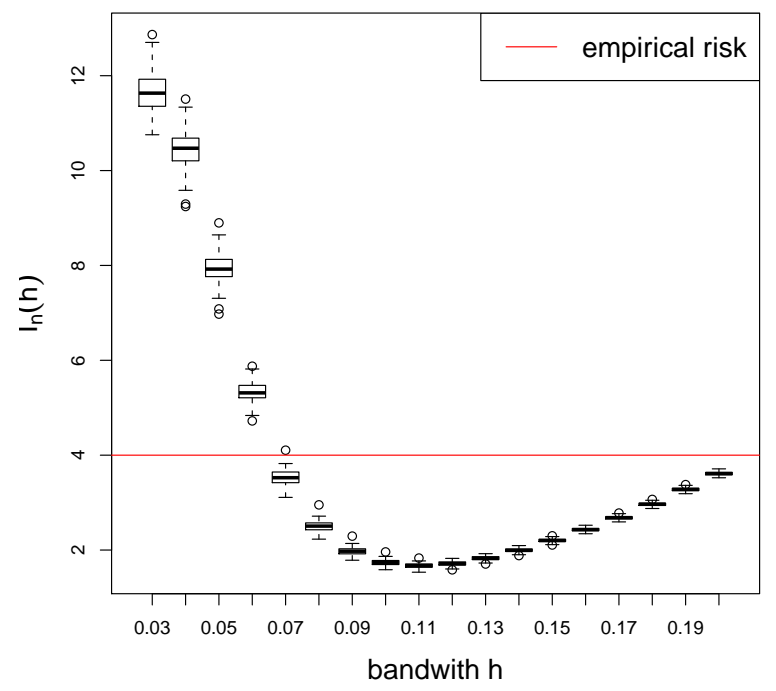

Figure 1: Boxplots of $I_{n}(h)$ for different bandwidth $h$. These results correspond to $N_{s}=100$ computations of the deconvolution estimator based on samples of size $n=20000$.

\begin{tabular}{|c|cccc|}
\hline$n$ & 30 & 100 & 500 & 1000 \\
$\hat{h}_{*}$ & 0.172 & 0.159 & 0.143 & 0.137 \\
$\bar{I}_{n}\left(\hat{h}_{*}\right)$ & $4.7 \mp 0.2$ & $3.8 \mp 0.2$ & $3.14 \mp 0.05$ & $2.78 \mp 0.05$ \\
\hline \hline$n$ & 5000 & 7500 & 10000 & 20000 \\
$\hat{h}_{*}$ & 0.123 & 0.119 & 0.117 & 0.111 \\
$\bar{I}_{n}\left(\hat{h}_{*}\right)$ & $2.12 \mp 0.02$ & $1.98 \mp 0.02$ & $1.87 \mp 0.02$ & $1.67 \mp 0.01$ \\
\hline
\end{tabular}

Table 1: Estimations of $\hat{h}_{*}$ and estimated risks for several values of the sample size $n$. These results have been computed thanks to $N_{s}=100$ computations of the deconvolution estimator.

corresponding estimation $\bar{I}_{n}\left(\hat{h}_{*}\right)$ of $\mathbb{E}\left(I_{n}\left(h_{*}\right)\right)$. For $n=7500, \bar{I}_{n}\left(\hat{h}_{*}\right)$ is about one half of $\mathbb{E}\left(W^{2}\left(\mu_{n}, \delta_{0}\right)\right)$.

Figure 2 shows a linear relation between $\log \hat{h}_{*}$ and $\log n$. A linear regression leads to an estimation of the slope of $-0.067=-1 / 14.9$, which is close (a little larger) to the theoretical slope: $-1 / 14$ (see Section 2.6. Case 2: isotropic Laplace noise with $d=2$ ).

As pointed out in Remark 3 of Section 2.4, it seems that $h_{*}$ does not strongly depend on the geometric shape $G$. Hence, the bandwidth $\hat{h}_{*}$ computed for the Dirac measure should be a reasonable bandwidth for estimating other distributions $\mu$ when the error distribution is an isotropic Laplace noise. This intuition is confirmed via the simulations presented in the next section. 


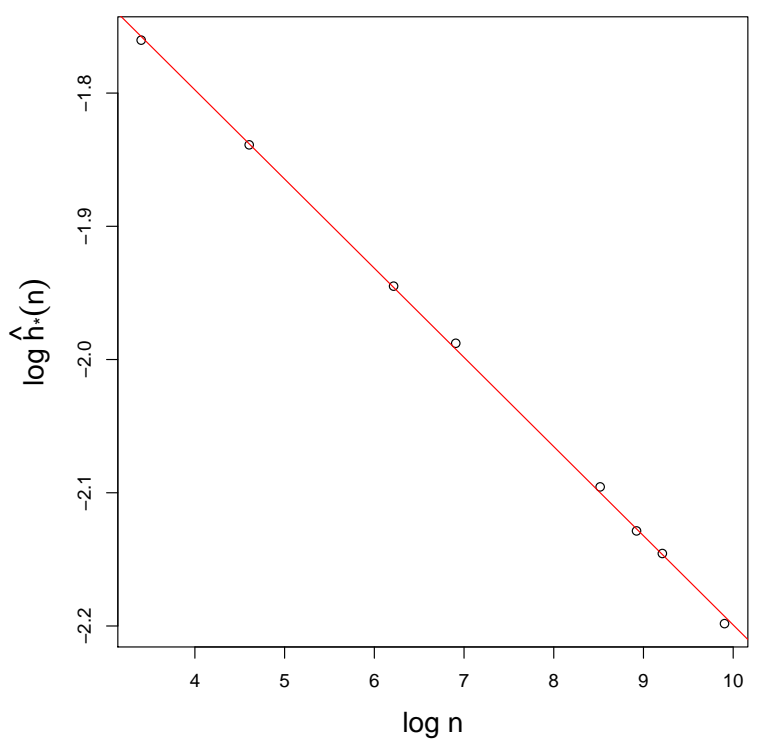

Figure 2: Estimation of the bandwidths $\hat{h}_{*}(n)$ (left) against the sample size in logarithm scales. The estimated slope for the regression of $\log \hat{h}_{*}(n)$ by $\log n$ is $-0.067 \approx-1 / 14.9$.

\subsection{Geometric inference}

This section illustrates with some simulations how to take advantage from the estimator $\hat{\mu}_{n}$ and its consistency properties for geometric inference purposes. As already explained in the introduction, the geometry of the unknown object $G$ can be inferred thanks to the levels of the distance function to a measure $\mathrm{d}_{\nu, m_{0}}$ defined by (1) if $\nu$ is close enough to $\mu$ for the Wasserstein metric. The following simulations compare the geometry recovered from the distance $\mathrm{d}_{\mu_{n}, m_{0}}$ to the empirical measure as in [4], and the distance $\mathrm{d}_{\hat{\mu}_{n}, m_{0}}$ to the deconvolution estimator $\hat{\mu}_{n}$. The scale parameter $m_{0}$ is fixed to $m_{0}=0.01$ for all the computations of the section. Hence we shall note $\mathrm{d}_{\nu}$ for $\mathrm{d}_{\nu, m_{0}}$ in the sequel.

\section{Three disks and Laplace noise}

For this first example, we consider the geometric shape in $\mathbb{R}^{2}$ composed of three disks of radius one whose centers are at a distance $\frac{5}{2}$ of each other. A total set of 20000 points is sampled uniformly on these disks and observed with an isotropic Laplace noise, as in Section 3.1. Figure 3 allows us to compare the distance function to the empirical measure $\mu_{n}$ and the distance function to the estimator $\tilde{\mu}_{n}$ deduced from the deconvolution estimator. For the bandwidth, we take $h=\hat{h}_{*}=0.11$, where $\hat{h}_{*}$ has been computed in Section 3.1 for the Dirac measure (see Table 1).

The deconvolution allows us to enlarge the numbers of levels which recovers the three disks : only the levels of $\mathrm{d}_{\mu_{n}}$ between 0.29 and 0.5 have the correct topology whereas the levels between 0.16 and 0.57 are valid for $\mathrm{d}_{\tilde{\mu}_{n}}$. Further- 


\section{Distance to the empirical measure}

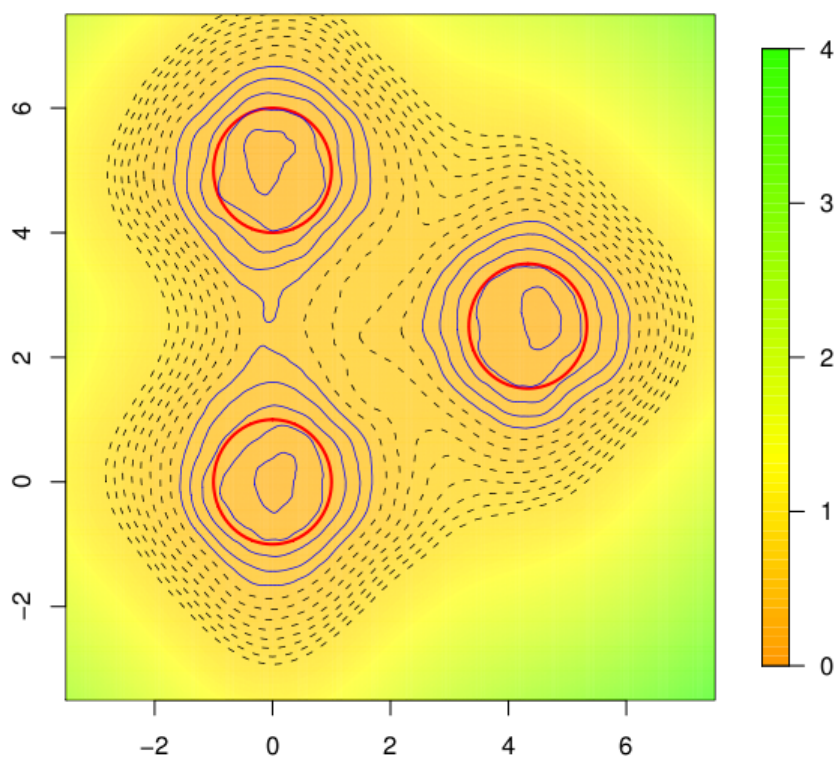

Distance to the estimator

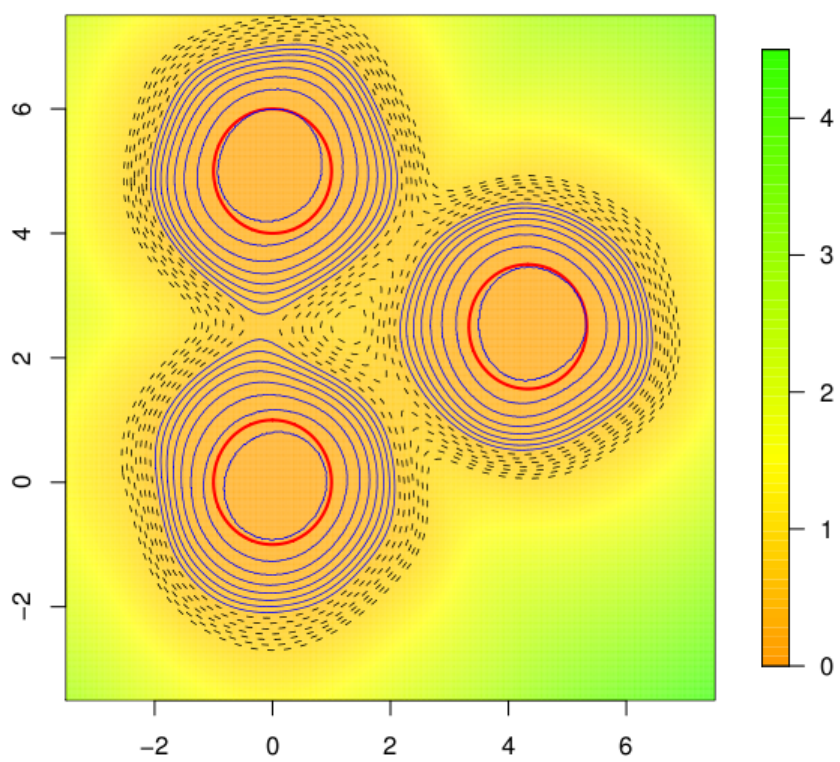

Figure 3: Distance $\mathrm{d}_{\mu_{n}}$ to the empirical measure and distance $\mathrm{d}_{\tilde{\mu}_{n}(0.11)}$ to the estimator for the three disks experiment with Laplace noise. The three circles delimiting the disks are drawn in red and the levels of the distance function which have the correct topology are drawn in blue. The other levels are the klack.dashed lines. The same grid of levels is used on the two pictures. 
more, by drawing and comparing the levels of $\mathrm{d}_{\tilde{\mu}_{n}(h)}$ for different bandwidth $h$, it can be checked that $h=0.11$ is around the optimal topological bandwidth, namely it corresponds to the larger scale of levels of correct topology.

\section{Two circles and Laplace noise}

The geometric shape of this second experiment is composed of two circles of radius 4 and 7 . A total set of 20000 points is sampled uniformly on these two circles and the sample is observed with an isotropic Laplace noise, as in Section 3.1. The benefit of using a deconvolution estimator is obvious in this context, since no levels of $\mathrm{d}_{\mu_{n}}$ can reach the correct topology, whereas the levels of $\mathrm{d}_{\tilde{\mu}_{n}}$ between 0.56 and 0.63 give the correct topology, see Figure 4. The bandwidth used here is again $h=\hat{h}_{*}=0.11$, as calibrated in Section 3.1

\section{One Gaussian example}

As explained in Section 2.6 a Gaussian noise will give a logarithmic rate of convergence of the deconvolution estimator $\hat{\mu}_{n}$ : this makes the application more difficult in this framework. Anyway, a Gaussian example is proposed here, but we use a large sample to be able to observe the topological effects.

The geometric shape to be recovered is composed of two embedded closed filaments. One set of $n=100000$ points are uniformly sampled on these two filaments and this sample is observed with a standard isotropic Gaussian noise, which means that $\varepsilon_{1,1}$ and $\varepsilon_{1,2}$ have a standard normal distribution. The two filaments are drawn on the two pictures of Figure 5. No one of the drawn levels of $\mathrm{d}_{\mu_{n}}$ recovers the correct topology. In fact it can be checked by drawing the contour plot with a thiner resolution that only the levels between 1.04 and 1.06 have the correct topology. We use a bandwidth $h=0.12$ for our deconvolution estimator. A larger scale of levels of $\mathrm{d}_{\tilde{\mu}_{n}}$ between 0.72 and 0.91 allows us to recover the correct topology.

\section{One directional measurement error}

For this example, we take $\mu_{1}=\delta_{0}$ and $\mu_{2}^{*}(u)=\left(1+u^{2}\right)^{-1}$, which means that $\varepsilon_{1,1}=0$ and that $\varepsilon_{1,2}$ has a standard Laplace distribution.

A set of 10000 points is sampled uniformly along an incomplete circle, and then observed with this one directional Laplace noise. The top picture of Figure 6 shows the sample and the incomplete circle in red, and the bottom picture shows a sample drawn according to $\tilde{\mu}_{n, \mathbf{h}}$ : the hole is impossible to see on these contaminated data whereas it is whith the deconvolved measure. However, due to the oscillations of the deconvolution estimator (which is a well known drawback of these kind of estimators) a small amount of the mass appears at a large distance of the circle. Using the distance function $\mathrm{d}_{\tilde{\mu}_{n}, m_{0}}$ is then particularly appropriate there, because this distance will ignore these outliers provided $m_{0}$ is not too small. Figure 7 compares the distance to the empirical measure with the distance to the estimator: the hole can be recovered only by the levels of $\mathrm{d}_{\tilde{\mu}_{n}}$. The correct levels of $\mathrm{d}_{\tilde{\mu}_{n}}$ are between 0.31 and 0.57. The estimator $\tilde{\mu}_{n}$ has been computed here with the bandwidths $h_{1}=0.07$ and $h_{2}=0.25$, this choice leading to a correct inference of the geometric shape. 


\section{Distance to the empirical measure}

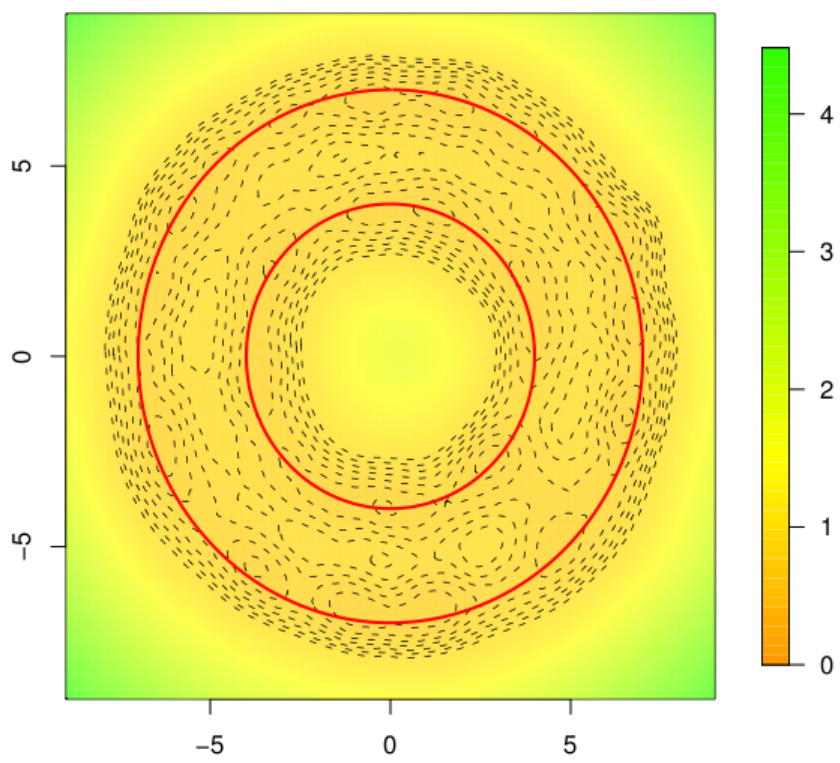

Distance to the estimator

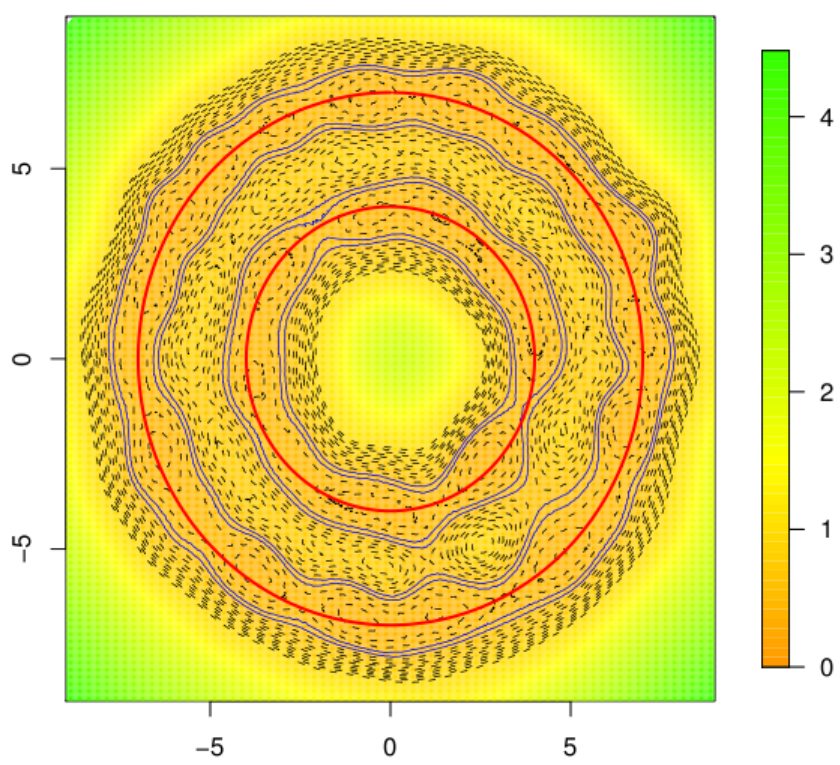

Figure 4: Distance $\mathrm{d}_{\mu_{n}}$ and distance $\mathrm{d}_{\tilde{\mu}_{n}(0.11)}$ for the two circles experiment with Laplace noise. See Figure 3 for more details about the legend. 

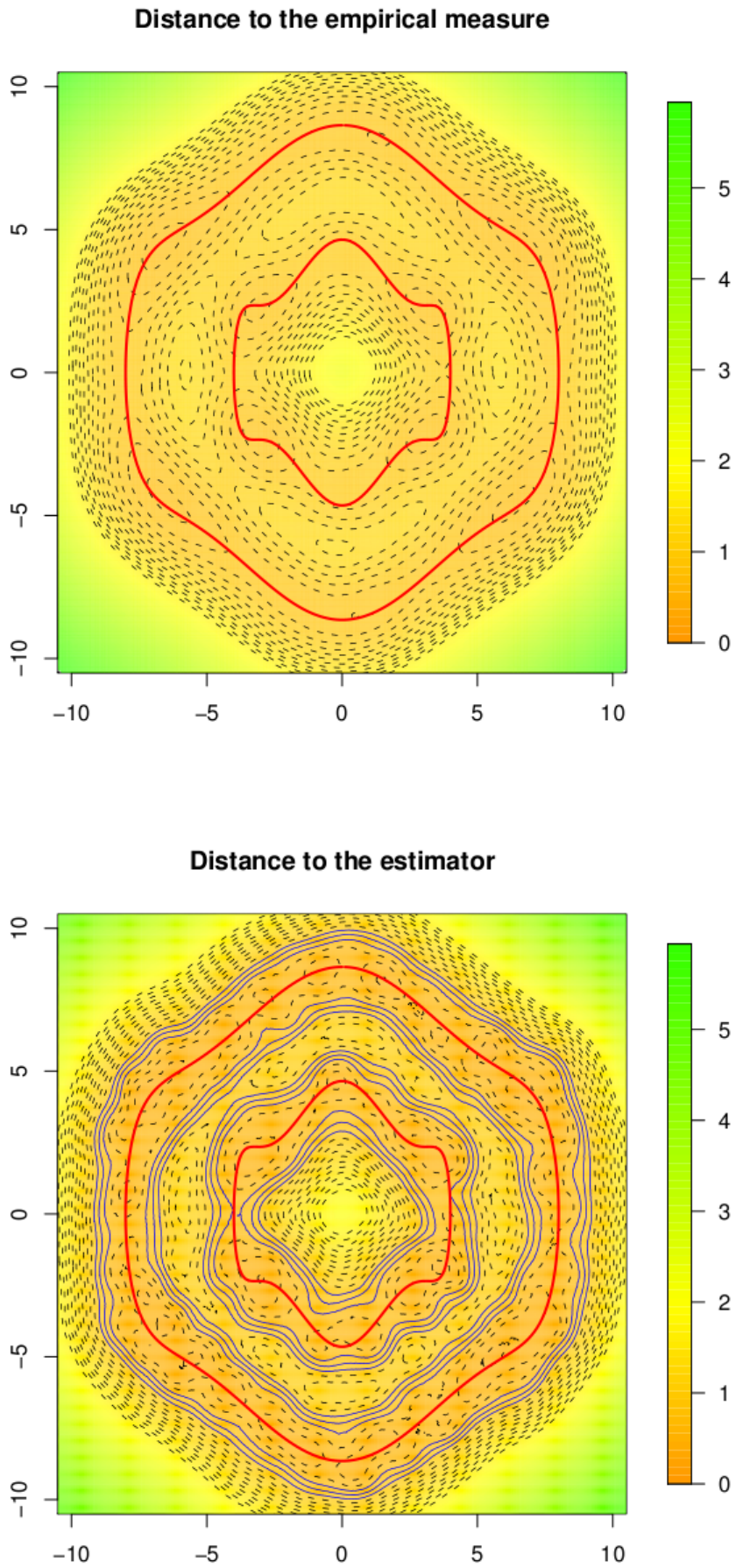

Figure 5: Distance $\mathrm{d}_{\mu_{n}}$ and distance $\mathrm{d}_{\tilde{\mu}_{n}(0.12)}$ for the two filaments experiment with Gaussian noise. See Figure 3 for more details about the legend. 

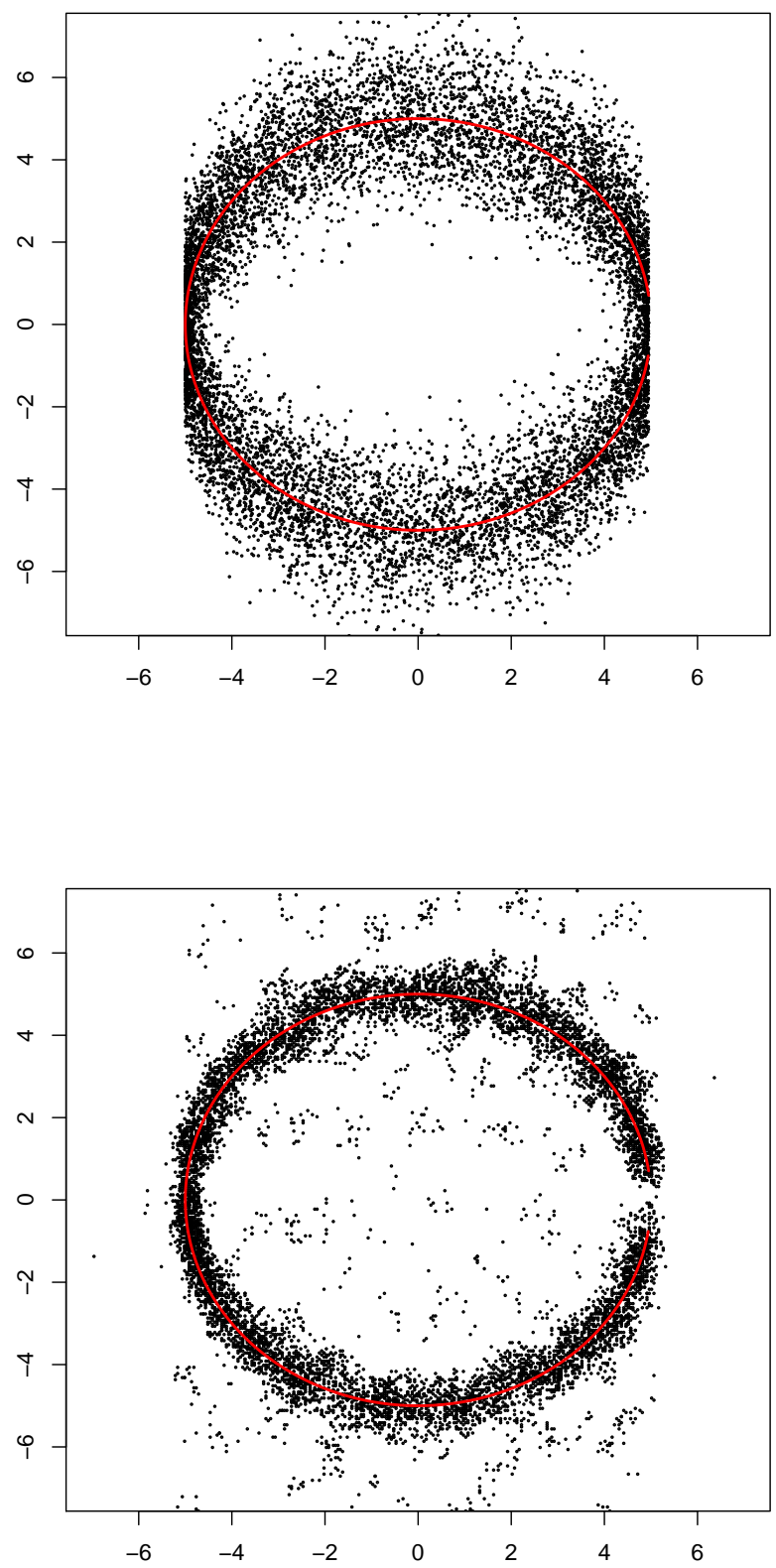

Figure 6: Circle with hole in red and 10000 points sampled on it with an unidirectional Laplace measurement error (top) and simulation of 10000 points according to $\tilde{\mu}_{n, \mathbf{h}}$ with $h_{1}=0.07$ and $h 2=0.25$ (bottom). 
Distance to the empirical measure

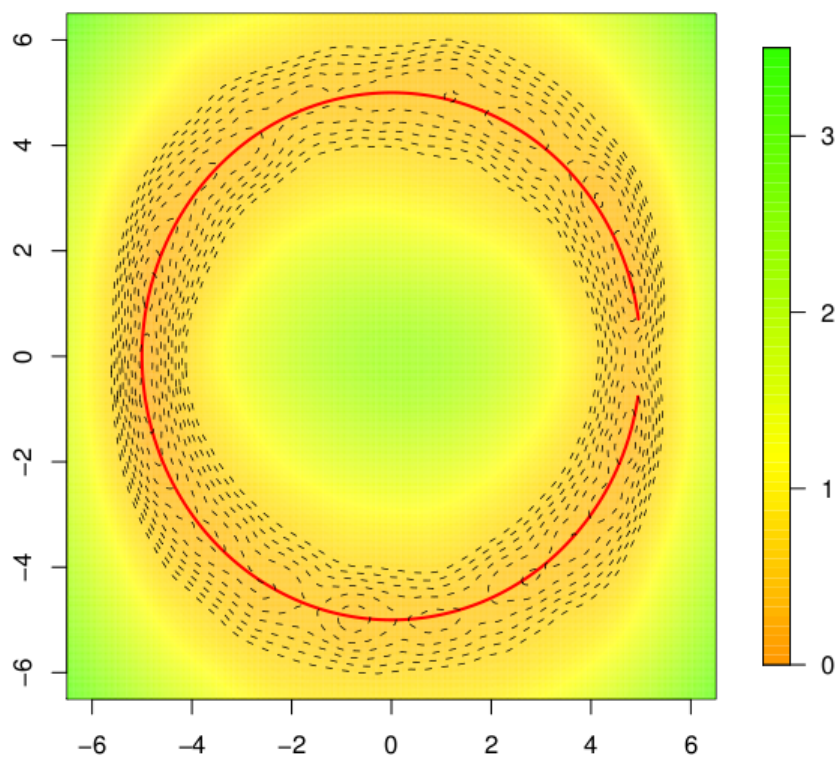

Distance to the estimator

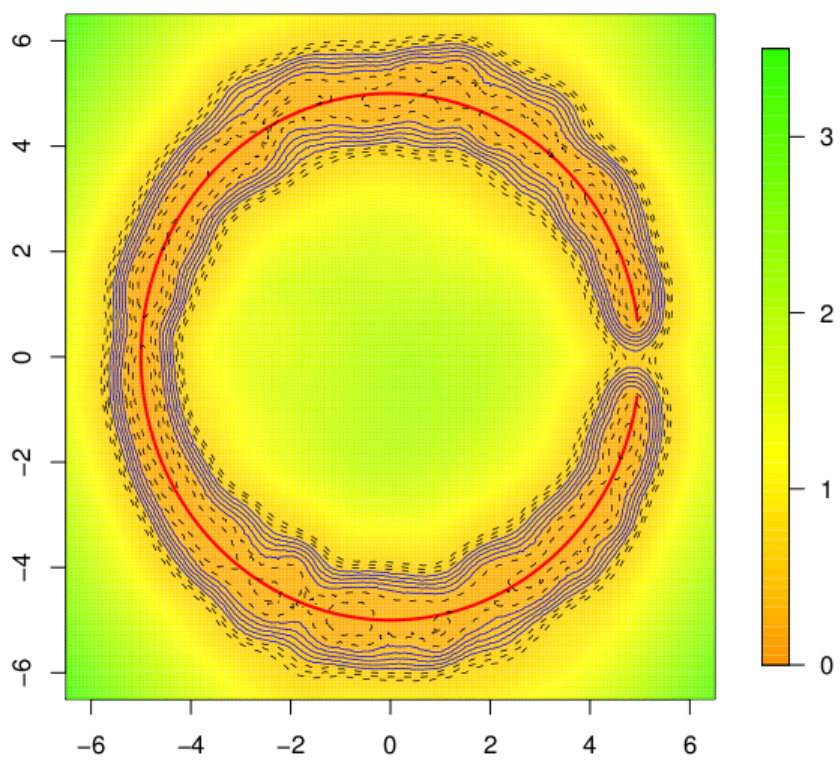

Figure 7: Distance $\mathrm{d}_{\mu_{n}}$ and distance $\mathrm{d}_{\tilde{\mu}_{n}}$ for the circle with hole with unidirectionnal noise. See Figure 3 for more details about the legend. 


\begin{tabular}{|c|c|}
\hline$k_{1}(x):=\sin (x) / x$ & $k_{1}^{*}(t)=1_{[-1,1]}$ \\
$k_{2}(x):=48(\cos x)\left(1-15 x^{-2}\right)\left(\pi x^{4}\right)^{-1}$ & $k_{2}^{*}(t)=\left(1-t^{2}\right)^{3} 1_{[-1,1]}$ \\
$k_{3}(x):=k(x)$, see 13 & $k_{3}^{*}=k^{*}$, see 13 \\
$k_{4}(x):=(2 \pi)^{-1 / 2} \exp \left(-x^{2} / 2\right)$ & $k_{4}^{*}(t)=\exp \left(-x^{2} / 2\right)$ \\
\hline
\end{tabular}

Table 2: Four kernels and their Fourier transform.

\section{A Kernel performances}

The section shortly discusses the kernel choice by comparing the performances of the deconvolution estimator for the most used kernels. In the density estimation framework with $\mathbb{L}_{2}$ risk in dimension one, 11] compare the performances of four kernels given in Table 2 .

Only $k_{3}$ fulfills all the required conditions (see Section 2.2 to prove the consistency result. Nevertheless, note that $k_{1}^{*}, k_{2}^{*}$ have a compact support $[-1,1]$. $k_{2}^{*}$ is also $C^{2}$ and its second derivate is Lipschitz, so this second kernel nearly fulfills the required assumptions. On the other hand $k_{1}^{*}$ is the less regular, and concerning the Gaussian kernel, $k_{4}^{*}$ has the required regularity but it has not a compact support.

The four kernels are compared in the simple situation in $\mathbb{R}$ for which the Wasserstein distance can be computed, namely a Dirac mass at 0. For each simulation, we consider a set of $n=500$ independent Laplace variables of variance 2. An accurate grid $\mathcal{P}$ of points $p$ over $\mathbb{R}$ is fixed, and for each simulation and a given bandwidth $h$, let $I_{n}(h)$ be the Wasserstein distance between $\delta_{0}$ and $\tilde{\mu}_{n}(h): I_{n}(h):=\sum_{p \in \mathcal{P}} x_{i}^{2} \hat{\alpha}_{i}(h)$. The Wasserstein risk is then estimated by computing $\bar{I}_{n}(h)$ over 100 simulations of this experience.

It appears that the two kernels $k_{1}$ and $k_{4}$ have very bad performances. Even for their estimated minimal bandwidth $\hat{h}_{*}$, the mean risk of their estimator is over 6 . This first observation tends to confirm that the kernel assumptions of Section 2.2 are not too restrictive. On the other hand, Figure 8 shows that $k_{2}$ and $k_{3}$ lead to estimators whose performance are quite similar in this context. This is not surprising since these two kernels have similar regularity properties. In spite of this observation, note that the consistency result is not proved for $k_{2}$ since it is not positive, and thus the control of the bias proposed in this paper is not valid for this kernel.

\section{References}

[1] Gérard Biau, Benoît Cadre, and Bruno Pelletier. Exact rates in density support estimation. J. Multivariate Anal., 99(10):2185-2207, 2008.

[2] Raymond J. Carroll and Peter Hall. Optimal rates of convergence for deconvolving a density. J. Amer. Statist. Assoc., 83(404):1184-1186, 1988.

[3] F. Chazal, D. Cohen-Steiner, and A. Lieutier. A sampling theory for compact sets in euclidean spaces. Discrete Comput Geom, 41:461-479, 2009. 


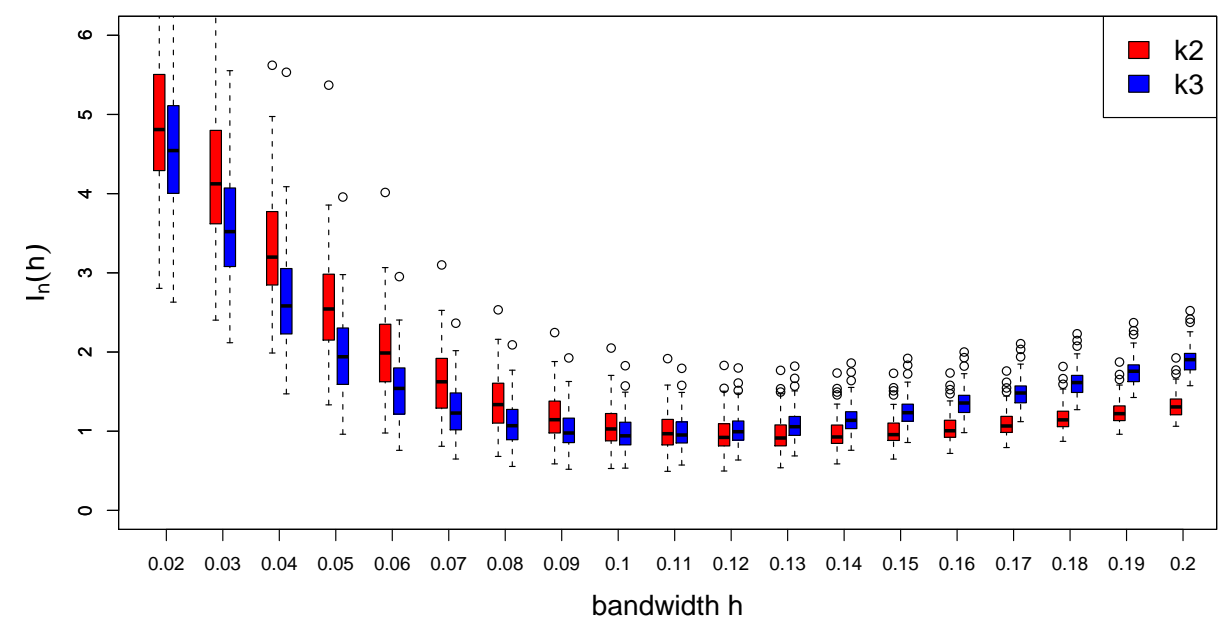

Figure 8: Comparing the performances of the deconvolution estimators defined by the two kernels $k_{2}$ and $k_{3}$.

[4] F. Chazal, D. Cohen-Steiner, and Q. Mérigot. Geometric inference for probability measures. J. Foundations of Computational Mathematics. to appear.

[5] F. Chazal and A. Lieutier. Smooth manifold reconstruction from noisy and non uniform approximation with guarantees. Comp. Geom: Theory and Applications, 40:156-170, 2008.

[6] Fabienne Comte and Claire Lacour. Anisotropic adaptive kernel deconvolution. hal-00579608, 2011.

[7] Antonio Cuevas, Manuel Febrero, and Ricardo Fraiman. Estimating the number of clusters. Canad. J. Statist., 28(2):367-382, 2000.

[8] Antonio Cuevas and Ricardo Fraiman. Set estimation. In New perspectives in stochastic geometry, pages 374-397. Oxford Univ. Press, Oxford, 2010.

[9] Antonio Cuevas, Ricardo Fraiman, and Alberto Rodríguez-Casal. A nonparametric approach to the estimation of lengths and surface areas. Ann. Statist., 35(3):1031-1051, 2007.

[10] A. Delaigle and I. Gijbels. Estimation of boundary and discontinuity points in deconvolution problems. Statist. Sinica, 16(3):773-788, 2006.

[11] Aurore Delaigle and Peter Hall. On optimal kernel choice for deconvolution. Statist. Probab. Lett., 76(15):1594-1602, 2006.

[12] Luc Devroye. Consistent deconvolution in density estimation. Canad. J. Statist., 17(2):235-239, 1989. 
[13] Christopher R. Genovese, Marco Perone-Pacifico, Isabella Verdinelli, and Larry Wasserman. On the path density of a gradient field. Ann. Statist., $37(6 \mathrm{~A}): 3236-3271,2009$.

[14] Christopher R. Genovese, Marco Perone-Pacifico, Isabella Verdinelli, and Larry Wasserman. The geometry of nonparametric filament estimation. arXiv:1003.5536v2, 2010.

[15] Peter Hall and Léopold Simar. Estimating a changepoint, boundary, or frontier in the presence of observation error. J. Amer. Statist. Assoc., 97(458):523-534, 2002.

[16] John A. Hartigan. Clustering algorithms. John Wiley \& Sons, New YorkLondon-Sydney, 1975. Wiley Series in Probability and Mathematical Statistics.

[17] Trevor Hastie and Werner Stuetzle. Principal curves. J. Amer. Statist. Assoc., 84(406):502-516, 1989.

[18] Joseph Horowitz and Rajeeva L. Karandikar. Mean rates of convergence of empirical measures in the Wasserstein metric. J. Comput. Appl. Math., $55(3): 261-273,1994$.

[19] V. I. Koltchinskii. Empirical geometry of multivariate data: a deconvolution approach. Ann. Statist., 28(2):591-629, 2000.

[20] A. Meister. Deconvolution problems in nonparametric statistics. Lecture Notes in Statistics 193. Springer-Verlag, 2009.

[21] Alexander Meister. Estimating the support of multivariate densities under measurement error. J. Multivariate Anal., 97(8):1702-1717, 2006.

[22] Alexander Meister. Support estimation via moment estimation in presence of noise. Statistics, 40(3):259-275, 2006.

[23] P. Niyogi, S. Smale, and S. Weinberger. A topological view of unsupervised learning from noisy data. SIAM Journal on Computing, 40(3):646-663, 2011.

[24] A. Petrunin. Semiconcave functions in Alexandrov's geometry. In Surveys in differential geometry. Vol. XI, pages 137-201. Int. Press, Somerville, MA, 2007.

[25] Svetlozar T. Rachev. Probability metrics and the stability of stochastic models. Wiley Series in Probability and Mathematical Statistics: Applied Probability and Statistics. John Wiley \& Sons Ltd., Chichester, 1991.

[26] Svetlozar T. Rachev and Ludger Rüschendorf. Mass transportation problems. Vol. II. Probability and its Applications. Springer-Verlag, 1998.

[27] Leonard Stefanski and Raymond J. Carroll. Deconvoluting kernel density estimators. Statistics, 21(2):169-184, 1990.

[28] C. Villani. Topics in Optimal Transportation. American Mathematical Society, Providence, 2003. 
[29] V. M. Zolotarev. Pseudomoments. Teor. Verojatnost. i Primenen., 23(2):284-294, 1978.

\section{Contents}

\begin{tabular}{lll}
\hline 1 & Introduction & 3
\end{tabular}

2 Deconvolution for the Wasserstein metric 5

2.1 The multivariate convolution model . . . . . . . . . . . . 6

2.2 Deconvolution estimators . . . . . . . . . . . . . . . . 6

2.3 A general decomposition . . . . . . . . . . . . . . . . . . . 7

2.4 Errors with independent coordinates . . . . . . . . . . . . . 10

2.5 Linear transform of errors with independent coordinates . . . . . 13

2.6 Examples of rates of convergence . . . . . . . . . . . . . 14

3 Experiments $\mathbf{1 5}$

$3.1 \quad$ Dirac experiment and bandwidth selection. . . . . . . . . . . . 16

3.2 Geometric inference .................. . . 18

\begin{tabular}{|l|l|}
\hline A Kernel performances & 25
\end{tabular} 
Centre de recherche INRIA Saclay - Île-de-France

Parc Orsay Université - ZAC des Vignes

4, rue Jacques Monod - 91893 Orsay Cedex (France)

Centre de recherche INRIA Bordeaux - Sud Ouest : Domaine Universitaire - 351, cours de la Libération - 33405 Talence Cedex Centre de recherche INRIA Grenoble - Rhône-Alpes : 655, avenue de l'Europe - 38334 Montbonnot Saint-Ismier

Centre de recherche INRIA Lille - Nord Europe : Parc Scientifique de la Haute Borne - 40, avenue Halley - 59650 Villeneuve d'Ascq Centre de recherche INRIA Nancy - Grand Est : LORIA, Technopôle de Nancy-Brabois - Campus scientifique 615, rue du Jardin Botanique - BP 101 - 54602 Villers-lès-Nancy Cedex

Centre de recherche INRIA Paris - Rocquencourt : Domaine de Voluceau - Rocquencourt - BP 105 - 78153 Le Chesnay Cedex

Centre de recherche INRIA Rennes - Bretagne Atlantique : IRISA, Campus universitaire de Beaulieu - 35042 Rennes Cedex

Centre de recherche INRIA Sophia Antipolis - Méditerranée : 2004, route des Lucioles - BP 93 - 06902 Sophia Antipolis Cedex 